\title{
Analysis of a Singular Convection Diffusion System Arising in Turbulence Modelling
}

\author{
P. Dreyfuss \\ Laboratoire J.A. Dieudonné, Université de Nice Sophia Antipolis, Parc Valrose, 06108 Nice, France \\ Correspondence should be addressed to P. Dreyfuss; dreyfuss@unice.fr
}

Received 16 April 2013; Accepted 7 July 2013

Academic Editor: Athanasios N. Yannacopoulos

Copyright (C) 2013 P. Dreyfuss. This is an open access article distributed under the Creative Commons Attribution License, which permits unrestricted use, distribution, and reproduction in any medium, provided the original work is properly cited.

We shall study some singular stationary convection diffusion system governing the steady state of a turbulence model closely related to the $k-\varepsilon$ one. We shall establish existence, positivity, and regularity results in a very general framework.

\section{Introduction}

We shall first recall some basic ideas concerning the statistical turbulence modelling for fluids. The reader can consult $[1,2]$ for a more detailed introduction.

Let $\mathbf{u}, p, \rho$, and $T$ be the velocity, pressure, density, and temperature of a Newtonien compressible fluid. Let also $\widetilde{\Omega} \subset$ $\mathbb{R}^{3}$ be a domain which is assumed to be bounded. Then the motion of the flow in $\widetilde{\Omega}$ at a time $t \in \mathbb{R}^{+}$can be described by the compressible Navier Stokes equations (see system (C) page 8 in [3]). It is well known that direct simulation based on such a model is harder or even impossible at high Reynolds numbers. The reason is that too many points of discretization are necessary, and so only very simple configurations can be handled.

Thus, engineers and physicists have proposed new sets of equations to describe the average of a turbulent flow. The most famous one is the $k-\varepsilon$ model, introduced by Kolmogorov [4]. We shall briefly present its basic principles in the following. Let $v$ denote a generic physical quantity subject to turbulent (i.e., unpredictable at the macroscopic scale); we introduce its mean part (or its esperance) $\langle v\rangle$ by setting:

$$
\langle v(x, t)\rangle=\int_{\mathscr{P}} v(\omega, x, t) d \mathscr{P}(\omega),
$$

where the integral is taken in a probablistic context which we shall not detail any more here. Note, however, that the operation $\langle\cdot\rangle$ is more generally called a filter. The probablistic meaning is one but not the only possible filter (see, for instance, [1] chapter 3). We shall then consider the decomposition: $v=\langle v\rangle+v^{\prime}$, where $v^{\prime}$ is referred to the noncomputable or the nonrelevant part and $\langle v\rangle$ is called the mean part (i.e., the macroscopic part).

The principle of the $k-\varepsilon$ model is to describe the mean flow in terms of the mean quantities $\langle\mathbf{u}\rangle,\langle p\rangle,\langle\rho\rangle$, and $\langle T\rangle$ together with two scalar functions $k$ and $\varepsilon$, which contains relevant information about the small scales processes (or the turbulent processes). The variable $k$ (SI: $\left.\left[\mathrm{m}^{2} / \mathrm{s}^{2}\right]\right)$ is called the turbulent kinetic energy, and $\varepsilon\left[\mathrm{m}^{2} / \mathrm{s}^{2}\right]$ is the rate of dissipation of the kinetic energy. They are defined by

$$
k=\frac{1}{2}\left|\mathbf{u}^{\prime}\right|^{2}, \quad \varepsilon=\frac{v}{2}\left\langle\left|\nabla \mathbf{u}^{\prime}+\left(\nabla \mathbf{u}^{\prime}\right)^{T}\right|^{2}\right\rangle
$$

where $v$ is the molecular viscosity of the fluid. The model is then constructed by averaging (i.e., by appling the operator $\langle\cdot\rangle$ on) the Navier-Stokes equations. Under appropriate assumptions (i.e., the Reynolds hypothesis in the incompressible case and the Favre average in the compressible case), we obtain a closed system of equations for the variables $\langle\mathbf{u}\rangle,\langle p\rangle,\langle\rho\rangle,\langle T\rangle$, $k$, and $\varepsilon$ (see [1] pages 61-62 for the incompressible case and pages 116-117 in the compressible situation).

Here, we shall focus on the equations for $k$ and $\varepsilon$, and we consider that the others quantities $\langle\mathbf{u}\rangle,\langle p\rangle,\langle\rho\rangle$, and $\langle T\rangle$ are known. Moreover, in order to simplify the readability, we do not use the notation $\langle\cdot\rangle$; that is, in the sequel we will write $\mathbf{u}$ instead of $\langle\mathbf{u}\rangle$ and $\rho$ instead $\langle\rho\rangle$ to represent the mean 
velocity and density of the fluid. The equations for $k$ and $\varepsilon$ are of convection-diffusion-reaction type:

$$
\begin{aligned}
\partial_{t} k & +\mathbf{u} \cdot \nabla k-\frac{c_{v}}{\rho} \operatorname{div}\left(\left(\nu+\rho \frac{k^{2}}{\varepsilon}\right) \nabla k\right) \\
& =c_{\nu} \frac{k^{2}}{\varepsilon} F-\frac{2}{3} k D-\varepsilon \\
\partial_{t} \varepsilon & +\mathbf{u} \cdot \nabla \varepsilon-\frac{c_{\varepsilon}}{\rho} \operatorname{div}\left(\left(\nu+\rho \frac{k^{2}}{\varepsilon}\right) \nabla \varepsilon\right) \\
& =c_{1} k F-\frac{2 c_{1}}{3 c_{v}} \varepsilon D-c_{2} \frac{\varepsilon^{2}}{k},
\end{aligned}
$$

where $D(x, t):=\operatorname{div} \mathbf{u}(x, t), F(x, t):=(1 / 2)\left|\nabla \mathbf{u}+(\nabla \mathbf{u})^{T}\right|^{2}-$ $(2 / 3) D(x, t)^{2} \geq 0$ (see Appendix B in the appendices), and $c_{\gamma}, c_{\varepsilon}, c_{1}$, and $c_{2}$ are generally taken as positive constants (see (A.3) in the appendices).

Note that (3) is only valid sufficiently far from the walls. In fact, in the vicinity of the walls of the domain $\widetilde{\Omega}$, there is a thin domain $\Sigma$ called logarithmic layer in which the modulus of the velocity goes from 0 to $\mathcal{O}(1)$. In this layer we can use some wall law or a one equation model (see [1] chap. 1 and [5]) instead of (3). However, (3) can be considered even in the logarithmic layer if we allow the coefficients $c_{\nu}, c_{\varepsilon}, c_{1}$, and $c_{2}$ to depend appropriately on some local Reynolds numbers (see [1, pages 59-60 and page 115]). In this last situation the system is called Low-Reynolds number $k-\varepsilon$ model.

In the following, we focus on the study in the domain $\Omega:=$ $\widetilde{\Omega} \backslash \Sigma$, and we assume that its boundary $\partial \Omega$ is Lipschitz (see [6] page 127). We denote by $\mathbf{n}(x)$ the outward normal defined for almost all points $x \in \partial \Omega$. The boundary conditions for $k$ and $\varepsilon$ on $\partial \Omega$ are then on the form

$$
k=k_{0}, \quad \varepsilon=\varepsilon_{0} \quad \text { on } \partial \Omega,
$$

where $k_{0}$ and $\varepsilon_{0}$ are strictly positive functions which can be calculated by using a wall law (see [1, page 59]) or a one equation model (see [5]). In the following, we assume that $k_{0}$ and $\varepsilon_{0}$ are given. Moreover, we can assume (see again [1, page 59]) that

$$
\mathbf{u} \cdot \mathbf{n}=0 \quad \text { on } \partial \Omega
$$

We shall concentrate in this paper on a modified system obtained after introducing the new variables $\theta[\mathrm{s}]$ and $\varphi\left[\mathrm{m}^{-2}\right]$ given by

$$
\theta=\frac{k}{\varepsilon}, \quad \varphi=\frac{\varepsilon^{2}}{k^{3}} .
$$

These variables have a physical meaning (see [2]): $\theta$ represents a characteristic time of turbulence and $L=\varphi^{-1 / 2}$ is a characteristic turbulent, length scale. By using this change of variable in (3) and after considering some modelisation arguments for the diffusion processes (see the appendices), we obtain

$$
\begin{aligned}
& \partial_{t} \theta+\mathbf{u} \cdot \nabla \theta-\frac{1}{\rho} \operatorname{div}\left(\left(\nu+\frac{c_{\theta} \rho}{\theta \varphi}\right) \nabla \theta\right) \\
&=-c_{3} \theta^{2} F+c_{4} \theta D+c_{5},
\end{aligned}
$$

$$
\begin{aligned}
\partial_{t} \varphi & +\mathbf{u} \cdot \nabla \varphi-\frac{1}{\rho} \operatorname{div}\left(\left(\nu+\frac{c_{\varphi} \rho}{\theta \varphi}\right) \nabla \varphi\right) \\
& =-\varphi\left(c_{6} \theta F-c_{7} D+c_{8} \theta^{-1}\right)
\end{aligned}
$$

where the coefficients $c_{\theta}, c_{\varphi}$, and $c_{i}$ are all positive.

Problem $(P)$ differs from the $k-\varepsilon$ one only by the diffusive parts, and it is attractive by some stronger mathematical properties. Another model closely related to these systems, and having some popularity, is the $k-\omega$ one (with $\omega=\theta^{-1}$; see for instance [2]).

In the papers $[7,8]$, the authors have established the existence of a weak solution for $(P)$ and a property of positiveness. This last feature takes the model useful in practice: it can be used directly or also as an intermediary stabilization procedure to the $k-\varepsilon$ one (see [9]). Another important property attempted for a turbulence model is its capability to predict the possible steady states. In the previous works (except in [10]) only the evolutive version of $(P)$ was studied under very restrictive assumptions. In [10], however, the stationary problem is studied, but it is simplified by considering a perturbation of the viscosities that artificially cancel the singularity of the system.

Hence, in this paper we shall study the stationary version of $(P)$ on a bounded domain $\Omega \subset \mathbb{R}^{N}, N=2$ or 3 , on which we impose the boundary conditions $\theta=a, \varphi=b$ on $\partial \Omega$. Remark that, by using (4) together with (6), we obtain

$$
a=\frac{k_{0}}{\varepsilon_{0}}, \quad b=\frac{\varepsilon_{0}^{2}}{k_{0}^{3}} .
$$

Hence, we can assume that $a$ and $b$ are strictly positive given functions.

We shall establish existence, positivity, and regularity results in a very general framework.

\section{Main Results}

2.1. Assumptions and Notations. Let $(Q)$ denote the stationary system associated with $(P)$. For simplicity we introduce the new parameters $C_{\text {ind }}:=\rho c_{\text {ind }}$ where the subscript "ind" takes the integer values $3,4,5,6,7$, and 8 or the letters $\theta$ and $\varphi$. Then, our main model $(Q)$ has the following form:

$$
\begin{gathered}
\rho \mathbf{u} \cdot \nabla \theta-\operatorname{div}\left(\left(\nu+\frac{C_{\theta}}{\theta \varphi}\right) \nabla \theta\right) \\
=-C_{3} F \theta^{2}+C_{4} \theta D+C_{5} \quad \text { in } \Omega, \\
\rho \mathbf{u} \cdot \nabla \varphi-\operatorname{div}\left(\left(\nu+\frac{C_{\varphi}}{\theta \varphi}\right) \nabla \varphi\right) \\
=-\varphi\left(C_{6} \theta F-C_{7} D+C_{8} \theta^{-1}\right) \quad \text { in } \Omega, \\
\theta=a, \quad \varphi=b \quad \text { on } \partial \Omega .
\end{gathered}
$$

For physical reasons we are only interested in positive solutions $(\theta, \varphi)$ for $(Q)$. Note, however, that even with 
this restriction, the problem $(Q)$ may be singular (i.e., the viscosities $v+\left(C_{\theta} / \theta \varphi\right)$ and $\nu+\left(C_{\varphi} / \theta \varphi\right)$ may be unbounded $)$. Moreover, because we allow $\nu \equiv 0$, the equations may degenerate (i.e., the viscosities may vanish). Hence, without additional restriction there may be various nonequivalent notions of weak solution (see for instance [11]).

In fact a good compromise between respect of the physics, simplification of the mathematical study, and obtention of significative results is to restrict $\theta$ and $\varphi$ to be within the class $\mathcal{S}$ defined by

$$
\begin{aligned}
\mathcal{S}=\left\{f: \Omega \longrightarrow \mathbb{R}^{+}\right. \text {such that } \\
\\
\left.\quad f \in H^{1}(\Omega) \cap L^{\infty}(\Omega), f^{-1} \in L^{\infty}(\Omega)\right\} .
\end{aligned}
$$

In particular, if the parameters appearing in $(Q)$ are sufficiently regular and if we restrict $\theta$ and $\varphi$ to be within the class $\mathcal{S}$, then the notion of a weak solution for (Q) is univocally defined: it is a distributional solution $(\theta, \varphi)$ that satisfies the boundary conditions in the sense of the trace.

In this last situation we will tell that $(\theta, \varphi)$ is a weak solution of $(Q)$ in the class $\mathcal{S}$.

In order to be able to consider such a weak solution for $(Q)$, we shall precise in the following some sufficient conditions of regularity for the data.

Let $N=2$ or 3 denote the dimension of the domain $\Omega$, and let $r$ be a fixed number such that

$$
r>\frac{N}{2} \text {. }
$$

We then have the following continuous injection (see Lemma 5):

$$
L^{r}(\Omega) \subset W^{-1, \beta}(\Omega), \quad \text { where } \beta=r^{*}>N .
$$

Recall that $D=\operatorname{div}(\mathbf{u})$. We will consider the following assumptions.

(i) Assumptions on $\Omega$ :

$$
\Omega \subset \mathbb{R}^{N} \text { is open, }
$$

bounded, and it has a Lipschitz boundary $\partial \Omega$.

(ii) Assumptions on the flow data (when $N=2$, one assumption in (14) can be relaxed: $\mathbf{u} \in\left(L^{p}(\Omega)\right)^{2}$ with $p>2$ (instead of $p=3$ ) is sufficient, but this would not improve any result significantly.) $\mathbf{u}, F, D, \rho$, and $v$ are as follows:

$$
\begin{gathered}
v \geq 0, \\
F, \rho: \Omega \longrightarrow \mathbb{R}^{+}, \quad \rho, \rho^{-1} \in L^{\infty}(\Omega), \quad F \in L^{r}(\Omega), \\
\mathbf{u} \in\left(L^{3}(\Omega)\right)^{N}, \quad D \in L^{r}(\Omega), \quad \operatorname{div}(\rho \mathbf{u})=0, \\
\mathbf{u} \cdot \mathbf{n}=0 \quad \text { on } \partial \Omega .
\end{gathered}
$$

(iii) Assumptions on the turbulent quantities on the boundary are as follows:

$$
\begin{gathered}
a, b \in H^{1 / 2}(\partial \Omega) \cap L^{\infty}(\partial \Omega), \\
a(x), b(x) \geq \delta>0 \quad \text { a.e. } x \in \partial \Omega,
\end{gathered}
$$

where $\delta>0$ is a fixed number. (iv) Assumptions on the model coefficients are as follows:

$$
\begin{gathered}
C_{i}: \Omega \longrightarrow \mathbb{R}^{+}, \quad C_{5}, C_{8} \in L^{r}(\Omega), \\
\text { for } i \neq 5,8: C_{i} \in L^{\infty}(\Omega), \\
C_{\text {ind }}: \Omega \times\left(\mathbb{R}^{+}\right)^{2} \longrightarrow \mathbb{R}^{+} \text {are Caratheodory, } \\
C_{\text {ind }}(x, v, w) \geq \alpha_{\text {ind }}>0 \quad \forall v, w \in \mathbb{R}^{+}, \text {for a.a. } x \in \Omega, \\
\forall v, w \in \mathbb{R}^{+}, \quad x \longrightarrow C_{\text {ind }}(x, v, w) \in L^{\infty}(\Omega),
\end{gathered}
$$

where in (18) $-(20) C_{\text {ind }}$ means $C_{\theta}$ or $C_{\varphi}$. The assumption (18) signifies that for all $v, w \in \mathbb{R}^{+}, x \rightarrow$ $C_{\text {ind }}(x, u, v)$ is measurable, and for a.a. $x \in \Omega$ : $(v, w) \rightarrow C_{\text {ind }}(x, v, w)$ is continuous. This ensures that $C_{\text {ind }}(x, \theta, \varphi)$ is measurable when $\theta$ and $\varphi$ are measurable. The condition (19) means that $C_{\text {ind }}$ is uniformly positive, whereas (20) tells that $C_{\text {ind }}(x, \theta, \varphi)$ remains bounded if $\theta$ and $\varphi$ are bounded.

We will study problem $(Q)$ under themain assumption:

$$
\left(A_{0}\right):(9)-(20) \text { are satisfied. }
$$

Note that in the main situation $\left(A_{0}\right)$ the assumption (12) made for $v$ allows the possibility that $v \equiv 0$. In other words the molecular viscosity $v$ can be neglected in the model. This is often chosen in practice because the eddy viscosities $C_{\theta} / \theta \varphi$ and $C_{\varphi} / \theta \varphi$ are dominant in the physical situations (see $\left.[1,5]\right)$. Remark also that the coefficients $C_{i}$ are allowed to depend on $x$, and the viscosity parameters $C_{\theta}, C_{\varphi}$ may depend on $x, \theta$, $\varphi$.

For a given function $f: \Omega \rightarrow \mathbb{R}$, we shall use the notations $f^{+}$and $f^{-}$to represent the positive and negative parts of $f$, that is

$$
\begin{gathered}
f=f^{+}+f^{-}, \\
f^{+}(x)=\max (f(x), 0) \geq 0, \\
f^{-}(x)=\min (f(x), 0) \leq 0 .
\end{gathered}
$$

We will also consider some assumptions of low compressibility of the form

$$
\left\|D^{+}\right\|_{L^{r}(\Omega)} \leq \tau,
$$

for some $\tau>0$ that will be precised.

This last kind of condition seems to be necessary (see the Appendices) in order to obtain a weak solution for (Q) in the three-dimensional case, whereas when $N=2$, we shall use some particularities of the situation to obtain a weak solution without any assumption of low compressibility. Nevertheless in this case we will assume that in addition to $\left(A_{0}\right)$ the following condition is satisfied:

$$
\left(A_{1}\right): \frac{C_{4}^{2}}{C_{3}} \in L^{r},
$$

$$
c_{6}=0.014, \quad c_{7}=0.104, \quad c_{8}=0.84 \text {. }
$$


In this last condition the values for $c_{6}, c_{7}$, and $c_{8}$ are in fact their classical constant values (see (A.8) in the Appendices)

In the sequel we denote by DATA some quantity depending only on the data under the assumption $\left(A_{0}\right)$, that is,

$$
\begin{gathered}
\text { DATA }=\text { Const }\left(\Omega, a, b,\|\mathbf{u}\|_{\left(L^{3}\right)^{N}}, \alpha_{\theta}, \alpha_{\varphi},\left(\left\|C_{j}\right\|_{L^{r}}\right)_{j=5,8},\right. \\
\left.\|F\|_{L^{r}},\left(\left\|C_{i}\right\|_{L^{\infty}}\right)_{i=3,4,6,7}\right) .
\end{gathered}
$$

Note that DATA does not depend on $D$ and $v$.

The exact form of the dependency (i.e., the function Const) is allowed to change from one part of the text to another.

2.2. Main Results. We shall establish two theorems of existence. The first one applies if $N=2$ or 3 , and the second one is limited to the case $N=2$.

Theorem 1. Assume that $\left(A_{0}\right)$ holds. Then, there exists $\tau>0$ such that, if $\left\|D^{+}\right\|_{L^{r}(\Omega)} \leq \tau$, then problem $(Q)$ admits at least one weak solution $(\theta, \varphi)$ in the class $\mathcal{S}$.

Theorem 2. Assume that $N=2$ and $\left(A_{0}\right),\left(A_{1}\right)$ hold. Then, problem $(Q)$ admits at least one weak solution $(\theta, \varphi)$ in the class $\mathcal{S}$. result.

In all the situations, we have the following regularity

Theorem 3. Let $(\theta, \varphi)$ be a weak solution of $(Q)$ in the class $\mathcal{S}$, and assume that $\left(A_{0}\right)$ is satisfied. We have the following.

(i) If $\mathbf{u} \in\left(L^{\infty}(\Omega)\right)^{N}$, and $a, b$ are Hölder continuous, then $(\theta, \varphi) \in\left(\mathscr{C}^{0, \alpha}(\bar{\Omega})\right)^{2}$, for some $\alpha>0$

(ii) Assume that in addition, the following conditions are satisfied:

$$
\begin{gathered}
\partial \Omega, \text { a and b are of class } \mathscr{C}^{2, \alpha}, \quad \rho \mathbf{u} \in\left(\mathscr{C}^{1, \alpha}(\bar{\Omega})\right)^{N}, \\
F \in \mathscr{C}^{0, \alpha}(\bar{\Omega}), \quad \forall i=3, \ldots, 8: C_{i} \in \mathscr{C}^{0, \alpha}(\bar{\Omega}), \\
C_{\text {ind }}(x, v, w) \in \mathscr{C}^{1, \alpha}\left(\bar{\Omega} \times\left(\mathbb{R}^{+}\right)^{2}\right) .
\end{gathered}
$$

Then $(\theta, \varphi) \in\left(\mathscr{C}^{2, \alpha}(\bar{\Omega})\right)^{2}$, and it is a classical solution of $(Q)$.

2.3. Discussion on the Results. Compared to previous works (see $[1,7,12,13])$ our basic assumption $\left(A_{0}\right)$ made in Theorem 1 is very general. In particular, we do not artificially cancel the singularity in the model, and we only assume weak regularity on the data. For instance, the basic assumption we made on the mean flow is $\mathbf{u} \in\left(L^{3}(\Omega)\right)^{N}$ and $\operatorname{div} \mathbf{u} \in L^{r}(\Omega)$ with some $r>3 / 2$, whereas in the previous works it was assumed that $\mathbf{u} \in\left(L^{\infty}(\Omega)\right)^{N}$ and $\operatorname{div} \mathbf{u} \in L^{\infty}(\Omega)$. Our condition is more interesting from a practical point of view because it is satisfied when $u$ is a weak solution of the Navier
Stokes equations. Hence, our work could be used for a future analysis of the full coupled system Navier-Stokes plus (Q).

From a mathematical point of view the problem we study is a nonlinear, degenerate, and singular elliptic system. Several complications arise for its analysis. In particular, the balance between the increase/decrease of the source terms (i.e., the functions $C_{i}, D$, and $F$ appearing in the second members of $(Q)$ ), and the possible explosion/vanishing of the viscosities is difficult to control. The strategy followed here is to first carefully study some elliptic scalar problem (possibly degenerate and singular) of the form

$$
\begin{gathered}
\rho \mathbf{u} \cdot \nabla \zeta-\operatorname{div}\left(\left(\nu+\frac{\kappa}{\zeta}\right) \nabla \zeta\right)=g(x, \zeta) \quad \text { in } \Omega, \\
\zeta=\zeta_{0} \quad \text { on } \partial \Omega .
\end{gathered}
$$

By using and developing some techniques due to Stampacchia, we are able to establish existence, positivity, and regularity results for problem $(S)$. These results (see Proposition 9), which also have an independent mathematical interest, are the key ingredients for proving Theorem 1 . Under the additional assumption $\mathbf{u} \in\left(L^{\infty}(\Omega)\right)^{N}$, we give a Hölder continuity result for $k$ and $\theta$. Moreover, we establish an existence result for a classical solution under some smoothness assumptions on the data.

\subsection{Organization of the Paper}

(i) In Section 3, we shall recall some results concerning the truncature at a fixed level and the Stampacchia's estimates. This last technique takes an important role in our analysis; moreover, we shall need a precise control of the estimates. Hence, we shall present it with some details and developments.

(ii) In Section 4 , we introduce a sequence $\left(Q_{n}\right)$ of problems which approximate $(Q)$. For $n$ fixed $\left(Q_{n}\right)$ is a PDE system of two scalar equations of the form $(S)$ : one equation for the unknown $\theta_{n+1}$ and one for $\varphi_{n+1}$. The point is that the unknowns $\theta_{n+1}$ and $\varphi_{n+1}$ are only weakly coupled. The coupling of the two equations is essentially realized through the quantities $\theta_{n}$ and $\varphi_{n}$ calculated at the previous step. Hence, we shall firstly study carefully the problem $(S)$. The major tool used here is the Stampacchia's estimates. We next prove that $\left(Q_{n}\right)$ is well posed. Hence, we obtain an approximate sequence of solutions $\left(\theta_{n}, \varphi_{n}\right)$ for problem (Q). Moreover, we prove that $\theta_{n}$ and $\varphi_{n}$ are uniformly bounded from above and below, which are the key estimates.

(iii) In Section 5, we use the uniform bounds established in Section 4, in order to extract a converging subsequence from $\left(\theta_{n}, \varphi_{n}\right)$. We then prove that the limit $(\theta, \varphi)$ is a weak solution of $(Q)$ in the class $\mathcal{S}$.

Under the additional assumption $\mathbf{u} \in\left(L^{\infty}(\Omega)\right)^{3}$, we are able to use the De Giorgi-Nash theorem, and we obtain an Hölder continuity result for $\theta, \varphi$. By assuming in addition some smoothness properties for 
the data, we can iterate the Schauder estimates and prove Theorem 3.

(iv) In the appendices, we present the derivation of the $\theta-\varphi$ model from the $k-\varepsilon$ one. Moreover, we justify that the choice $\varphi=\varepsilon^{2} / k^{3}$ is valid even in the compressible situation. The justification uses in particular a property of positivity of the function $F$. We also discuss briefly the necessity of the low compressibility assumption when $N=3$. Finally, we recall a generalized version of the chain rule for $G(u)$ where $G$ is a Lipchitz function and $u$ a Sobolev one.

\section{Mathematical Background}

In this section, we shall recall some results concerning the truncature at a fixed level and the Stampacchia's estimates. This last technique takes an important role in our analysis; moreover, we shall need a precise control of the estimates. Hence, we shall present here the technique with some details and developments. As in the rest of the paper we denote by $\Omega \subset \mathbb{R}^{N}$ a bounded open Lipschitz domain. These properties for $\Omega$ are always implicity assumed if they are not precised.

3.1. Truncatures and Related Properties. The technique of Stampacchia is based on the use of special test functions which are constructed by using some truncatures. We shall recall some basic properties of the truncatures used in the paper. An important tool is the generalized chain rule (see Theorem D.1 in the appendices).

Let $l>0$; we denote by $T_{l}$ the truncature function $T_{l}$ : $\mathbb{R} \rightarrow \mathbb{R}$ defined by

$$
T_{l}(s)= \begin{cases}l & \text { if } s>l \\ s & \text { if }-l \leq s \leq l \\ -l & \text { if } s<-l\end{cases}
$$

Let $v \in H^{1}(\Omega)$. By applying Theorem D.1, we see that $T_{l}(v) \in$ $H^{1}(\Omega) \cap L^{\infty}(\Omega)$. Moreover, if we denote by $\Omega_{v, l}$ the set $\{x \in$ $\Omega$ s.t. $|v(x)| \leq l\}$, then we have

$$
\nabla T_{l}(v)= \begin{cases}\nabla v & \text { in } \Omega_{v, l} \\ 0 & \text { elsewhere }\end{cases}
$$

Note that $T_{l}(\cdot)$ truncates both the positive and the negative large values. In some cases, we need only to truncate the positive or the neghative side. For this reason, we introduce the semitruncatures $T_{l,+}$ and $T_{l,-}$ defined by

$$
\begin{gathered}
T_{l,+}(t)= \begin{cases}l & \text { if } t>l \\
t & \text { elsewhere }\end{cases} \\
T_{l,-}(t)= \begin{cases}-l & \text { if } t<-l \\
t & \text { elsewhere. }\end{cases}
\end{gathered}
$$

We then have the decomposition: $T_{l}=T_{l,+} \circ T_{l,-}=T_{l,-} \circ T_{l,+}$.
For given $v_{l} \in H^{1}(\Omega)$ and $s>0$, we shall also consider

$$
\psi_{s}(v)=v_{l}-T_{s}\left(v_{l}\right)= \begin{cases}v_{l}-s & \text { in } A_{s, l}^{+} \\ v_{l}+s & \text { in } A_{s, l}^{-} \\ 0 & \text { elsewhere }\end{cases}
$$

where we have used the notations:

$$
\begin{gathered}
A_{s, l}^{+}:=\left\{v_{l} \geq s\right\}, \quad A_{s, l}^{-}:=\left\{v_{l} \leq-s\right\}, \\
A_{s, l}=A_{s, l}^{+} \cup A_{s, l}^{-} .
\end{gathered}
$$

Let also $\psi_{s, \pm}$ be the functions defined above (30) while replacing $T_{s}$ by $T_{s,+}$ or by $T_{s,-}$. It is easy to verify that $\psi_{s,+}$ (resp., $\psi_{s,+}$ ) is in fact the positive (resp., the negative) part of $\psi_{s}$. In other words, we have

$$
\begin{gathered}
\psi_{s,+}\left(v_{l}\right)=\psi_{s}^{+}\left(v_{l}\right)=\left(v_{l}-s\right) \mathbf{1}_{A_{s, l}^{+}} \geq 0, \\
\psi_{s,-}\left(v_{l}\right)=\psi_{s}^{-}\left(v_{l}\right)=\left(v_{l}+s\right) \mathbf{1}_{A_{s, l}^{-}} \leq 0 . \\
\psi_{s}=\psi_{s}^{+}+\psi_{s}^{-} .
\end{gathered}
$$

The function $\psi_{s}$ has the following properties:

Lemma 4. (i) $\psi_{s} \in H^{1}(\Omega)$ and $\nabla \psi_{s}=\mathbf{1}_{A_{s, l}} \nabla v_{l}$,

(ii) if $\gamma v_{l} \in L^{\infty}(\partial \Omega)$, then for all $s>\left\|\gamma v_{l}\right\|_{L^{\infty}(\partial \Omega)}$, we have $\psi_{s} \in H_{0}^{1}(\Omega)$.

Where $\gamma: H^{1}(\Omega) \rightarrow H^{1 / 2}(\partial \Omega)$ denotes the trace function.

Proof. Point (i) is a direct consequence of Theorem D.1. Property (ii) is proved in [14, Lemma 3.3 p. 53].

3.2. The Stampacchia Estimates. The Stampacchia estimates is a general method which allows one to obtain an $L^{\infty}$-estimate for the weak solution of a large class of elliptic PDEs of the second order. The $L^{\infty}$-estimate presented in the original work [15] depends on various quantities related to the PDE problem studied, but the exact dependency is not established. In our analysis we need a precise control of the $L^{\infty}$-estimates with respect to some quantities (in particular with respect to the diffusion coefficient of the PDEs). Hence, in the following, we take over and detail the technique in order to obtain a more precise $L^{\infty}$-estimates.

The Stampacchia estimates are established by using the test functions $\psi_{s}$ (or $\psi_{s}^{ \pm}$) defined previously, where in this case $v$ (resp., $v_{l}$ ) is a weak solution of the problem (resp., the sequence of problems) considered.

For technical reasons we need a classical result concerning some relationships between $L^{r}$ functions and linear form on Sobolev spaces.

Lemma 5. Let $1 \leq r<\infty$ and $E \in L^{r}(\Omega)$. Then

$$
E \in W^{-1, \beta}(\Omega), \quad \text { with } \beta=r^{*}=\frac{N r}{N-r},
$$


and there exists $\widetilde{E} \in\left(L^{\beta}(\Omega)\right)^{3}$ such that

$$
\begin{gathered}
\int_{\Omega} E \varphi=\int_{\Omega} \widetilde{E} \cdot \nabla \varphi \quad \forall \varphi \in \mathscr{D}(\Omega), \\
\|\widetilde{E}\|_{\left(L^{\beta}(\Omega)\right)^{3}} \leq\|E\|_{L^{r}(\Omega)} .
\end{gathered}
$$

Moreover, we have

$$
r>\frac{N}{2} \Longrightarrow \beta>N
$$

Proof. Property (33) is easy to prove by using the Sobolev injection Theorem; together with the Hölder inequality: $\varphi \rightarrow$ $\int_{\Omega} E \varphi$ is a linear form on $W_{0}^{1, p}$ if $p^{*}:=N p /(N-p) \geq r /(r-1)$. This last condition holds for $p=P:=N r /(r(N+1)-N)$. Hence, $P^{\prime}=p /(p-1)=N r /(N-r)=r^{*}$.

We next obtain (34) by using a classical result (see [16] Proposition IX.20).

Finally, if we assume that $r>N / 2$ then $\beta=r^{*}>$ $\left(N^{2} / 2\right) /(N-N / 2)=N$.

The Stampacchia technique works in two steps: the first one is dependent on the problem (or the sequence of problems) studied, and the second one is independent of it. Here, the purpose is to present the key ingredients of these two steps. Because the first one is dependent on the problem studied we cannot present it here in its entirety, but we will consider a simple problem which contains the main technical points (in fact this introductive presentation will be useful to treat a more complicated class of problems in Section 4). Let $\left(v_{l}\right) \subset H_{0}^{1}(\Omega)$ be a sequence of functions satisfying

$$
\int_{\Omega} v_{l} \nabla v_{l} \nabla \varphi=\int_{\Omega} g_{l} \varphi, \quad \forall \varphi \in H_{0}^{1},
$$

where $\left(\nu_{l}\right)$ is a given sequence of strictly positive bounded functions and $\left(g_{l}\right) \subset L^{r}(\Omega)$, with $r>N / 2$. Let also $m_{l}, M_{l}$ denote the bounds from above and below for $\nu_{l}$, that is,

$$
0<m_{l} \leq v_{l} \leq M_{l}<\infty, \quad \text { a.e. in } \Omega \text {. }
$$

Step 1. By testing (36) with $\psi_{s}=\psi_{s}\left(v_{l}\right)$, we obtain

$$
m_{l} \int_{\Omega}\left|\nabla \psi_{s}\right|^{2}=\int_{\Omega} g_{l} \psi_{s} \stackrel{\text { by Lemma } 5}{=} \int_{\Omega} E_{l} \nabla \psi_{s},
$$

with $E_{l} \in L^{\beta},\left\|E_{l}\right\|_{L^{\beta}} \leq\left\|g_{l}\right\|_{L^{r}}$ for some $\beta=\beta(r)>N$.

Recall also that Supp $\psi_{s}\left(v_{l}\right) \subset A_{l, s}$. Hence, by using the Hölder inequality we obtain

$$
\begin{gathered}
\int_{\Omega} E_{l} \nabla \psi_{s} \leq\left\|\nabla \psi_{s}\right\|_{L^{2}}\left(\int_{A_{l, s}}\left|E_{l}\right|^{2}\right)^{1 / 2} \\
\leq\left\|\nabla \psi_{s}\right\|_{L^{2}}\left\|E_{l}\right\|_{L^{\beta}}\left(\int_{A_{l, s}} 1\right)^{1 / 2(\beta / 2)^{\prime}} \\
\leq\left\|\nabla \psi_{s}\right\|_{L^{2}}\left\|E_{l}\right\|_{L^{\beta}}\left|A_{l, s}\right|^{(\beta-2) / 2 \beta} .
\end{gathered}
$$

Consequently (38) leads to

$$
\left\|\psi_{s}\right\|_{H_{0}^{1}(\Omega)^{2}}^{2} \leq C\left|A_{l, s}\right|^{\Phi}, \quad \text { with } C>0, \Phi>\frac{2}{2^{*}}=\frac{N-2}{N} .
$$

This is the key estimate needed to pass at the second step which is independent of the problem studied.

Note that with the particular sequence of problems (36) chosen here, the constants $C$ and $\Phi$ are

$$
C=\frac{\left\|g_{l}\right\|_{L^{r}}^{2}}{m_{l}^{2}}, \quad \Phi=\frac{\beta-2}{\beta} .
$$

Hence, $\Phi$ does not depend on $l, s$. Moreover, if we assume that $\left(g_{l}\right)$ is uniformly bounded in the $L^{r}$-norm and that $\left(m_{l}\right)$ is uniformly bounded from above by a strictly positive constant, then $C$ is also independent of $l, s$.

This is an important point because we will see it hereafter; an estimate (40) with $C$ and $\Phi$ independent of $l, s$ leads to a uniform $L^{\infty}$ bound for $\left(v_{l}\right)$.

Step 2. Assume that we have obtained (40). We can obtain an $L^{\infty}$-estimate for $v_{l}$ as follows.

Let $2^{*}=2 N /(N-2)$ be the Sobolev exponent associated to 2 in dimension N. By using the Poincaré-Sobolev inequality we have

$$
\left(\int_{A_{l, s}}\left|\psi_{s}\right|^{2^{*}}\right)^{2 / 2^{*}} \leq C_{1}\left\|\psi_{s}\right\|_{H_{0}^{1}(\Omega)}^{2}, \quad C_{1}=C_{1}(|\Omega|) .
$$

Let now $t>s$. It is clear that $A_{l, t} \subset A_{l, s}$, and consequently

$$
\begin{aligned}
\left(\int_{A_{l, s}}\left|\psi_{s}\right|^{2^{*}}\right)^{2 / 2^{*}} & \geq\left(\int_{A_{l, t}}\left|\psi_{s}\right|^{2^{*}}\right)^{2 / 2^{*}} \\
& \geq\left(\int_{A_{l, t}}|t-s|^{2^{*}}\right)^{2 / 2^{*}} \\
& \geq|t-s|^{2}\left|A_{l, t}\right|^{2 / 2^{*}}
\end{aligned}
$$

We set

$$
\chi(s):=\left|A_{l, s}\right|, \quad \forall s \geq 0 .
$$

For fixed $l, \chi$ is a decreasing function, and from the estimates (40)-(43), we obtain

$$
\begin{array}{r}
\chi(t) \leq C_{2}|\chi(s)|^{2^{*} \Phi / 2}(t-s)^{-2^{*}} \quad \forall t>s \geq 0, \\
C_{2}=\left(C_{1} C\right)^{2^{*} / 2} .
\end{array}
$$

Recall that we have assumed in (40) that $\Phi>2 / 2^{*}$. Hence, $2^{*} \Phi / 2>1$, and by using Lemma 4.1 in [15] we obtain

$$
\chi(d)=0, \quad d=2^{2^{*} \Phi /\left(2^{*} \Phi-2\right)} C_{2}^{1 / 2^{*}}|\Omega|^{(\Phi / 2)-\left(1 / 2^{*}\right)}<\infty .
$$

This property tells exactly that

$$
\left\|v_{l}\right\|_{L^{\infty}(\Omega)} \leq d, \quad d=C_{3}(\Phi, N,|\Omega|) \sqrt{C} .
$$


In particular, $d$ does not depend on $l$ if the constants $C$ and $\Phi$ appearing in (40) are independent of $l$. For instance with the particular sequence of problems (36) the constants $C$ and $\Phi$ are given by (41), and if we assume that $\left\|g_{l}\right\|_{L^{r}} \leq K, m_{l} \geq m>$ 0 , we obtain

$$
d=\widetilde{C} \frac{K}{m}, \quad \widetilde{C}=\widetilde{C}(\Omega, N, r)
$$

Remark 6. (i) If you are only interested in obtaining a uniform majoration or minoration for $v_{l}$ then instead of (40) it suffices to have

$$
\left\|\psi_{s}^{ \pm}\right\|_{H_{0}^{1}(\Omega)^{2}}^{2} \leq C^{ \pm}\left|A_{l, s}^{ \pm}\right|^{\Phi^{ \pm}}, \quad \text { with } C^{ \pm}>0, \Phi^{ \pm}>\frac{N-2}{N}
$$

In fact in this case we consider $\chi_{ \pm}(s):=\left|A_{l, s}^{ \pm}\right|$instead of (44). This function is decreasing, and we obtain again (46). But now this property tells that

$$
\pm v_{l}(x) \leq d^{ \pm} \quad \text { a.e. in } \Omega, d^{ \pm}=\widetilde{C}^{ \pm}\left(\Phi^{ \pm}, N,|\Omega|\right) \sqrt{C^{ \pm}}
$$

(ii) Let again $\left(v_{l}\right)$ be a sequence of functions satisfying (36), and assume that $m_{l} \geq m>0$. Then, we have

$$
\begin{gathered}
g_{l} \leq h_{l}, \quad H:=\sup _{l}\left\|h_{l}\right\|_{L^{r}}<\infty \Longrightarrow v_{l} \leq d_{1} \quad \text { a.e. in } \Omega, \\
g_{l} \geq k_{l}, \quad K:=\sup _{l}\left\|k_{l}\right\|_{L^{r}}<\infty \Longrightarrow v_{l} \geq-d_{2} \quad \text { a.e. in } \Omega, \\
\text { with } d_{1}, d_{2}>0, \quad d_{1}=\widetilde{C} \frac{H}{m}, \quad d_{2}=\widetilde{C} \frac{K}{m}, \\
\widetilde{C}=\widetilde{C}(\Phi, N,|\Omega|) .
\end{gathered}
$$

The proof of (51) and (52) is obtained by taking over the first step of the technique of Stampacchia: we use the test function $\psi_{s}^{ \pm}$instead of $\psi_{s}$.

In fact, the function $\psi_{s}^{+}$is positive. Hence, instead of (38), we have

$$
m \int_{\Omega}\left|\nabla \psi_{s}^{+}\right|^{2}=\int_{\Omega} g_{l} \psi_{s}^{+} \leq \int_{\Omega} h_{l} \psi_{s}^{+}=\int_{\Omega} E_{l,+} \nabla \psi_{s}^{+},
$$

with $E_{l,+} \in L^{\beta},\left\|E_{l,+}\right\|_{L^{\beta}} \leq\left\|h_{l}\right\|_{L^{r}} \leq H$. Consequently, in this case, we obtain (49) for the function $\psi_{s}^{+}$, with $C^{+}=H^{2} / m^{2}$, and the uniform majoration (51) follows.

The relation (52) can be proven by using $\psi_{s}^{-}$as test function in (36). In fact, we remark now that $\psi_{s}^{-}$is negative. Hence, instead of (38) we obtain

$$
m \int_{\Omega}\left|\nabla \psi_{s}^{-}\right|^{2}=\int_{\Omega} g_{l} \psi_{s}^{-} \leq \int_{\Omega} k_{l} \psi_{s}^{-}=\int_{\Omega} E_{l,-} \nabla \psi_{s}^{-}
$$

with $E_{l,-} \in L^{\beta},\left\|E_{l,-}\right\|_{L^{\beta}} \leq\left\|k_{l}\right\|_{L^{r}} \leq K$. Consequently, we now obtain (49) for the function $\psi_{s}^{-}$, with $C^{-}=K^{2} / m^{2}$. This implies $-v_{l} \leq d_{2}$, and consequently $v_{l} \geq-d_{2}$.

\section{Approximate Sequence and Estimates}

Let $g \in H^{1 / 2}(\partial \Omega)$; we denote by $\mathscr{R} g$ its harmonic lifting that is:

$$
\mathscr{R} g \in H^{1}(\Omega), \quad \mathscr{R} g=g \quad \text { on } \partial \Omega, \quad \Delta \mathscr{R} g=0 \quad \text { on } \Omega \text {. }
$$

We define the functions $\theta_{0}$ and $\varphi_{0}$ by the formula

$$
\theta_{0}:=\mathscr{R} a \quad \varphi_{0}:=\mathscr{R} b \text {. }
$$

Hence, by the using the maximum principle (see [16, p. 189] and [17]) together with the condition (16), we obtain

$$
0<\delta \leq \theta_{0} \leq\|a\|_{L^{\infty}(\partial \Omega)}, \quad \delta \leq \varphi_{0} \leq\|b\|_{L^{\infty}(\partial \Omega)} .
$$

Let now $n \geq 0,\left(\theta_{n}, \varphi_{n}\right)$ be given, and

$$
\begin{aligned}
& C_{\theta}^{(n)}(\cdot):=C_{\theta}\left(\cdot, \theta_{n}(\cdot), \varphi_{n}(\cdot)\right), \\
& C_{\varphi}^{(n)}(\cdot):=C_{\varphi}\left(\cdot, \theta_{n}(\cdot), \varphi_{n}(\cdot)\right) .
\end{aligned}
$$

In order to construct an approximate solution $\left(\theta_{n+1}, \varphi_{n+1}\right)$ for problem $(Q)$, we introduce the following system:

$$
\begin{gathered}
\rho \mathbf{u} \cdot \nabla \theta_{n+1}-\operatorname{div}\left(\left(\nu+\frac{C_{\theta}^{(n)}}{\theta_{n+1} \varphi_{n}}\right) \nabla \theta_{n+1}\right)=g_{\theta}^{(n)} \text { in } \Omega, \\
\rho \mathbf{u} \cdot \nabla \varphi_{n+1}-\operatorname{div}\left(\left(\nu+\frac{C_{\varphi}^{(n)}}{\theta_{n+1} \varphi_{n+1}}\right) \nabla \varphi_{n+1}\right)=g_{\varphi}^{(n)} \text { in } \Omega, \\
\theta_{n+1}=a, \quad \varphi_{n+1}=b \quad \text { on } \partial \Omega,
\end{gathered}
$$

where we used the notations

$$
\begin{gathered}
g_{\theta}^{(n)}:=C_{5}-C_{3} F \theta_{n+1}^{2}+C_{4} \theta_{n+1} D, \\
g_{\varphi}^{(n)}:=-\varphi_{n}\left(C_{8} \theta_{n+1}^{-1}+C_{6} \theta_{n+1} F-C_{7} D\right) .
\end{gathered}
$$

For $n \in \mathbb{N}$ we denote by $\left(H_{n}\right)$ the following condition:

$$
\begin{gathered}
\theta_{n}, \varphi_{n}, \theta_{n}^{-1}, \varphi_{n}^{-1} \in L^{\infty}(\Omega), \\
\theta_{n}, \varphi_{n} \geq 0 .
\end{gathered}
$$

Let $\varphi_{\max }$ be a fixed real number such that

$$
\varphi_{\max }>\|b\|_{L^{\infty}(\partial \Omega)} .
$$

We shall also consider the condition: $\left(K_{n}\right):=\left(H_{n}\right)+\left(\varphi_{n} \leq\right.$ $\left.\varphi_{\max }\right)$.

Note that (57) shows that the condition $\left(K_{n}\right)$ is satisfied for $n=0$. We will prove in the sequel that, under condition $\left(K_{n}\right)$, we can obtain a weak solution $\left(\theta_{n+1}, \varphi_{n+1}\right)$ for problem $\left(Q_{n}\right)$, with moreover $\left(\theta_{n+1}, \varphi_{n+1}\right)$ satisfying the condition $\left(K_{n+1}\right)$. This last property ensures the right definition of an approximate sequence. More precisely, we have the following. 
Proposition 7. Let $n \in \mathbb{N}$ be given, and assume that $\left(A_{0}\right)$ is satisfied. Let also $\left(\theta_{n}, \varphi_{n}\right)$ be given and satisfy condition $\left(K_{n}\right)$. There exists $\tau>0$ depending only on DATA such that if $\left\|D^{+}\right\|_{L^{r}(\Omega)} \leq \tau$, then problem $\left(Q_{n}\right)$ admits at least one weak solution $\left(\theta_{n+1}, \varphi_{n+1}\right) \subset\left(H^{1}(\Omega) \times L^{\infty}(\Omega)\right)^{2}$.

Moreover, $\left(\theta_{n+1}, \varphi_{n+1}\right)$ satisfies condition $\left(K_{n+1}\right)$ and the estimates

$$
\begin{aligned}
& 0<\theta_{\min } \leq \theta_{n+1} \leq \theta_{\max }, \\
& 0<\varphi_{\min } \leq \varphi_{n+1} \leq \varphi_{\max },
\end{aligned}
$$

where $\varphi_{\max }$ was fixed in (60) and $\varphi_{\min }, \theta_{\min }$, and $\theta_{\max }$ are positive numbers depending on DATA, but not on $n$.

Remark 8. Proposition 7 is the key result that will be used later on to prove Theorem 1, whereas for Theorem 2 we shall establish and use a more simple version of this proposition (see Section 5.2).

In order to prove the proposition we establish intermediate results.

4.1. Auxiliary Results. Let $n \in \mathbb{N}$, and let $\left(\theta_{n}, \varphi_{n}\right)$ be given and satisfy $\left(K_{n}\right)$. We want to obtain $\left(\theta_{n+1}, \varphi_{n+1}\right)$ by solving $\left(Q_{n}\right)$, and in order to iterate the algorithm we also want that $\left(\theta_{n+1}, \varphi_{n+1}\right)$ satisfies $\left(K_{n+1}\right)$.

Remark that the system $\left(Q_{n}\right)$ is composed of two coupled scalar elliptic equations in divergence form, with a possible singular and degenerate structure. Hence, the goal of this subsection is to study this last kind of scalar problem.

In order to do this, we first introduce a weight $\kappa: \mathbb{R}^{+} \rightarrow$ $\mathbb{R}^{+}$which is assumed to be measurable and satisfying

$$
0<\kappa_{0} \leq \kappa \leq \kappa_{1} \quad \text { a.e. in } \Omega,
$$

where $\kappa_{0}$ and $\kappa_{1}$ are two given reals.

Let also $g: \Omega \times \mathbb{R}^{+} \rightarrow \mathbb{R}$ be a Caratheodory function (i.e., for all $u \in \mathbb{R}^{+}: x \rightarrow g(x, u)$ is measurable, and for a.a. $x \in \Omega: u \rightarrow g(x, u)$ is continuous).

Let us consider the scalar problem $(S)$ defined in Section 2 after Theorem 1 , where $\zeta_{0} \in H^{1 / 2}(\partial \Omega) \cap L^{\infty}(\partial \Omega), \zeta_{0} \geq \delta>0$ a.e. in $\delta \Omega$, is given. We always assume that $\rho, \mathbf{u}, \nu, \Omega$ which appear in $(S)$ satisfy their corresponding conditions in $\left(A_{0}\right)$.

Recall that we allow $v \equiv 0$ in $\left(A_{0}\right)$. Hence, problem $(S)$ may degenerate (i.e., the viscosity $\nu+(\kappa / \zeta)$ may vanish) when $\zeta \rightarrow \infty$. Moreover, $(S)$ is singular (i.e., the viscosity tends to infinity) when $\zeta \rightarrow 0$.

We want now to find sufficient additional conditions for $g$ that guarantee the existence of a bounded positive weak solution for problem $(S)$. Hence, we shall consider

$$
\begin{gathered}
\gamma(x):=\sup _{u \in[0,1]}\left|g^{-}(x, u)\right| \in L^{r}(\Omega), \\
g^{+}(x, u) \leq \gamma_{1}(x)+\gamma_{2}(x) h(u),
\end{gathered}
$$

where $\gamma_{1}, \gamma_{2} \in L^{r}(\Omega)$ and $h: \mathbb{R}^{+} \rightarrow \mathbb{R}^{+}$is continuous. In fact, more than establishing only the existence of a bounded positive solution for $(S)$, we are interested in obtaining some uniform (with respect to $\kappa_{1}$ ) bounds from above and below and some regularity results. We have the following.
Proposition 9. (i) Let $\kappa$ satisfy (62), and let $g: \Omega \times \mathbb{R}^{+} \rightarrow \mathbb{R}$ be a Caratheodory function satisfying (63), (64). There exists a real $\tau>0$ depending on $\kappa_{0}$, $h$ such that if $\left\|\gamma_{2}\right\|_{L^{r}(\Omega)} \leq \tau$, then there exists a weak solution $\zeta \in H^{1}(\Omega) \cap L^{\infty}(\Omega)$ for problem (S). Moreover, we have

$$
0<\zeta_{\min } \leq \zeta \leq \zeta_{\max }, \quad \zeta_{\min }=e^{-C / \kappa_{0}}, \zeta_{\max }=e^{C / \kappa_{0}},
$$

where $C$ depends only on $\gamma, \gamma_{1}, \Omega, r, N, \zeta_{0}$. In particular $\zeta_{\min }$ and $\zeta_{\max }$ are independent of $\kappa_{1}$.

In addition, the following extended (when $g^{+} \equiv 0$, it is a maximum principle) maximum principle holds:

$$
\begin{gathered}
\|\zeta\|_{L^{\infty}(\Omega)} \leq\left\|\zeta_{0}\right\|_{L^{\infty}(\partial \Omega)}+\widetilde{C} \frac{\left\|g^{+}\right\|_{L^{r}}}{\kappa_{0}}\|\zeta\|_{L^{\infty}(\Omega)}, \\
\widetilde{C}=\widetilde{C}\left(\Omega, N, r, \gamma_{1}\right) .
\end{gathered}
$$

(ii) Assume that in addition $\mathbf{u} \in\left(L^{\infty}(\Omega)\right)^{N}$ and $\zeta_{0}$ is Hölder continuous. Then, $\zeta \in \mathscr{C}^{0, \alpha}(\bar{\Omega})$ for some $0<\alpha<1$. Moreover, if $\partial \Omega$ is of class $\mathscr{C}^{2, \alpha}, g \in \mathscr{C}^{0, \alpha}\left(\bar{\Omega} \times \mathbb{R}^{+}\right)$, $\rho \mathbf{u} \in$ $\left(\mathscr{C}^{1, \alpha}(\bar{\Omega})\right)^{N}, \kappa \in \mathscr{C}^{1, \alpha}(\bar{\Omega})$, and $\zeta_{0} \in \mathscr{C}^{2, \alpha}(\partial \Omega)$, then $\zeta \in$ $\mathscr{C}^{2, \alpha}(\bar{\Omega})$, and it is a classical solution of $(S)$.

Before proving Proposition 9, we establish an intermediate result. In a first step we consider the change of variable $v=\ln \zeta$ in $(S)$, and for $l \in \mathbb{N}$ we introduce a truncated version $\left(S_{l}\right)$ of the system obtained:

$$
\begin{gathered}
\rho \mathbf{u} \nabla e^{T_{l}(v)}-\operatorname{div}\left(\left(v e^{T_{l}(v)}+\kappa\right) \nabla v\right) \\
=g\left(x, e^{T_{l}(v)}\right) \quad \text { in } \Omega, \\
v=\ln \zeta_{0} \quad \text { on } \partial \Omega .
\end{gathered}
$$

We then establish the following.

Lemma 10. (i) Let $\kappa$ satisfy (62), and let $g: \Omega \times \mathbb{R}^{+} \rightarrow \mathbb{R}$ be a Caratheodory function satisfying (63), (64). Then, for any $l \in \mathbb{N}$, there exists a weak solution $v=v_{l} \in H^{1}(\Omega) \cap L^{\infty}$ for the problem $\left(S_{l}\right)$.

(ii) Let $\left(v_{l}\right)$ be the sequence given in (i), and let $l \geq 1$ be a fixed integer. Then, there exists $\tau_{l}>0$ such that if the function $\gamma_{2}$ in (63) satisfies $\left\|\gamma_{2}\right\|_{L^{r}} \leq \tau_{l}$ then we have

$$
\left\|v_{l}\right\|_{L^{\infty}(\Omega)} \leq C \frac{\left\|\gamma+\gamma_{1}\right\|_{L^{r}}}{\kappa_{0}}, \quad C=C\left(\text { DATA }, \zeta_{0}\right) .
$$

In particular, $C$ is independent of $\kappa, v$, and $l$.

Proof. (i) By using the divergence formula, we obtain, for all $w \in H^{1}(\Omega)$,

$$
\begin{aligned}
\int_{\Omega} \rho \mathbf{u} \nabla e^{T_{l}(v)} \cdot w \\
=-\int_{\Omega} e^{T_{l}(v)} \operatorname{div}(\rho \mathbf{u} w)+\underbrace{\int_{\partial \Omega} e^{T_{l}(v)} \rho w \mathbf{u} \cdot \mathbf{n} d \sigma}_{=0} \\
=-\int_{\Omega} e^{T_{l}(v)} \rho \mathbf{u} \cdot \nabla w-\int_{\Omega} e^{T_{l}(v)} \underbrace{\operatorname{div}(\rho \mathbf{u})}_{=0} w .
\end{aligned}
$$


Let $v_{0}:=\ln \left(\mathscr{R} \zeta_{0}\right)$, and consider the change of variable $\widetilde{v}:=v-v_{0}$. Then, problem $\left(S_{l}\right)$ is equivalent to find $\widetilde{v} \in H_{0}^{1}(\Omega)$ such that

$$
-\operatorname{div} \sigma(x, \widetilde{v}, \nabla \widetilde{v})=f(x, \widetilde{v}) \quad \text { in } \mathscr{D}^{\prime}(\Omega),
$$

where $\sigma: \Omega \times \mathbb{R} \times \mathbb{R}^{N} \rightarrow \mathbb{R}^{N}$ and $f: \Omega \times \mathbb{R} \rightarrow \mathbb{R}$ are defined by

$$
\begin{gathered}
\sigma(x, w, \mathbf{G})=\left(\nu e^{T_{l}\left(w+v_{0}\right)}+\kappa(x)\right) \mathbf{G}-\rho(x) \mathbf{u}(x) e^{T_{l}\left(w+v_{0}(x)\right)}, \\
f(x, w)=g\left(x, e^{T_{l}\left(w+v_{0}(x)\right)}\right) .
\end{gathered}
$$

We now remark that (69) is a quasilinear equation in divergence form. Moreover, it is easy to see that $f$ and $\sigma$ satisfy the classical growth assumptions and $\sigma$ satisfies also the classical coercivity condition. Note that

$$
\begin{aligned}
& \left\langle\sigma(x, w, \mathbf{G})-\sigma\left(x, w, \mathbf{G}^{\prime}\right), \mathbf{G}-\mathbf{G}^{\prime}\right\rangle \\
& \quad=\left(\nu e^{T_{l}\left(w+v_{0}\right)}+\kappa\right)\left|\mathbf{G}-\mathbf{G}^{\prime}\right|^{2} \\
& \quad \geq \kappa_{0}\left|\mathbf{G}-\mathbf{G}^{\prime}\right|^{2} .
\end{aligned}
$$

Hence, $\sigma$ is strictly monotonous in the third variable. We then conclude (see for instance [18, Theorem 1.5] or [14, Theorem 8.8 page 311]) that there exists a weak solution $\widetilde{v} \in$ $H_{0}^{1}(\Omega)$ for $(69)$.

Consequently, $v_{l}:=\widetilde{v}+v_{0}$ is a weak solution for $\left(S_{l}\right)$, that is, for all $w \in H_{0}^{1}(\Omega)$,

$\int_{\Omega}\left(\nu e^{T_{l}\left(v_{l}\right)}+\kappa\right) \nabla v_{l} \nabla w+\int_{\Omega} \rho \mathbf{u} \nabla e^{T_{l}\left(v_{l}\right)} w=\int_{\Omega} g\left(x, e^{T_{l}\left(v_{l}\right)}\right) w$.

By applying Theorem 4.2 page 108 in [15], we obtain $v_{l} \epsilon$ $L^{\infty}(\Omega)$.

(ii)

$$
\text { Let } \tau_{l}:=\frac{1}{h\left(e^{l}\right)}>0 \text {, and assume now that }\left\|\gamma_{2}\right\|_{L^{r}} \leq \tau_{l} \text {. }
$$

With this additional assumption, we are able to obtain a useful estimation for $\left\|v_{l}\right\|_{L^{\infty}}$. Technically, we will detail a method due to Stampacchia. (See Section 3.2 for the notations and for an introduction of the method. Here only the first step of the technique will be developed further). Let $s>\left|\ln \left\|\zeta_{0}\right\|_{L^{\infty}(\partial \Omega)}\right|$; we consider the function $\psi_{s}=v_{l}-T_{s}\left(v_{l}\right)$. We have (see Lemma 4) $\psi_{s} \in H_{0}^{1}(\Omega) \cap L^{\infty}(\Omega)$, and by testing (72) with $\psi_{s}$, we obtain

$$
\begin{aligned}
\int_{\Omega}\left(\nu e^{T_{l}\left(v_{l}\right)}+\kappa\right)\left|\nabla \psi_{s}\right|^{2} \\
\quad+\underbrace{\int_{\Omega} \rho \mathbf{u} \cdot \nabla e^{T_{l}\left(v_{l}\right)} \psi_{s}}_{=: \mathrm{I}}=\underbrace{\int_{\Omega} g\left(x, e^{T_{l}\left(v_{l}\right)}\right) \psi_{s} .}_{=: \mathrm{II}}
\end{aligned}
$$

(a) We will now evaluate the terms I and II.

The term I is simplified by writing one of its integrand factors, namely, $\nabla e^{T_{l}\left(v_{l}\right)} \psi_{s}$, as a gradient. More precisely we have $\nabla e^{T_{l}\left(v_{l}\right)} \psi_{s}=\nabla \zeta_{l}$, with $\zeta_{l} \in H^{1}(\Omega) \cap L^{\infty}$ (see Lemma D.2 in the Appendices). Hence, by applying the divergence formula, we see that I vanishes:

$$
\mathrm{I}=\int_{\Omega} \rho \mathbf{u} \cdot \nabla \zeta_{l} \stackrel{\text { div. formula }}{=}-\int_{\Omega} \zeta_{l} \underbrace{\operatorname{div} \rho \mathbf{u}}_{=0}+\underbrace{\int_{\partial \Omega} \rho \zeta_{l} \mathbf{u} \cdot \mathbf{n} d \sigma}_{=0 \text { by }(15)}=0 .
$$

We next estimate the term II:

$$
\begin{aligned}
\mathrm{II} & =\int_{\Omega} g\left(x, e^{T_{l}\left(v_{l}\right)}\right) \psi_{s} \\
& \leq \underbrace{\int_{A_{s, l}^{+}} g^{+}\left(x, e^{T_{l}\left(v_{l}\right)}\right) \psi_{s}}_{:=\mathrm{II}_{1}}+\underbrace{\int_{A_{s, l}^{-}} g^{-}\left(x, e^{T_{l}\left(v_{l}\right)}\right) \psi_{s}}_{:=\mathrm{II}_{2}} .
\end{aligned}
$$

Remark now that on $A_{s, l}^{-}$we have $v_{l} \leq-s \leq 0$, which implies that $e^{T_{l}\left(v_{l}\right)} \leq 1$. Consequently by using the assumption (63), we obtain

$$
\mathrm{II}_{2} \leq \int_{\Omega} \gamma \psi_{s} \mathbf{1}_{A_{s, l}^{-}} .
$$

The term $\mathrm{II}_{1}$ is majorated as follows:

$$
\mathrm{II}_{1} \underset{\text { by (64) }}{\leq} \int_{A_{s, l}^{+}}\left(\gamma_{1}+\gamma_{2} h\left(e^{l}\right)\right) \psi_{s} \underset{\text { by }(73)}{\leq} \int_{\Omega}\left(\gamma_{1}+1\right) \psi_{s} \mathbf{1}_{A_{s, l}^{+}} .
$$

(b) At this point by using the estimates (75), (77), and (78) together with (74) and (62), we obtain

$$
\kappa_{0} \int_{\Omega}\left|\nabla \psi_{s}\right|^{2} \leq \int_{\Omega} \underbrace{\left(\gamma \mathbf{1}_{A_{s, l}^{-}}+\left(\gamma_{1}+1\right) \mathbf{1}_{A_{s, l}^{+}}\right)}_{=: E} \psi_{s} .
$$

Note that $E \in L^{r}(\Omega)$ and $\|E\|_{L^{r}} \leq C_{0}, C_{0}=\|\gamma\|_{L^{r}}+\left\|\gamma_{1}+1\right\|_{L^{r}}$. On the other hand (see Lemma 5) there exists $\widetilde{E} \in\left(L^{\beta}(\Omega)\right)^{3}$ satisfying $\|\widetilde{E}\|_{\left(L^{\beta}\right)^{N}} \leq\|E\|_{L^{r}}$, and $\int_{\Omega} E \varphi=\int_{\Omega} \widetilde{E} \nabla \varphi$ for all $\varphi \epsilon$ $H_{0}^{1}$. Recall also that we have assumed in (9) that $r>N / 2$ which implies $\beta>N$. By again using the Hölder inequality, we obtain for $\varphi \in H_{0}^{1}$

$$
\int_{\Omega} \widetilde{E} \nabla \varphi \leq\left.\|\widetilde{E}\|_{\left(L^{\beta}\right)^{N}}\|\varphi\|_{H_{0}^{1}} \operatorname{Supp} \varphi\right|^{(\beta-2) / 2 \beta} .
$$

Consequently, (79) leads to:

$$
\begin{aligned}
& \kappa_{0}\left\|\psi_{s}\right\|_{H_{0}^{1}}^{2} \\
& \leq \int_{\Omega} \widetilde{E} \nabla \psi_{s} \leq C_{0}\left|A_{s, l}\right|^{(\beta-2) / 2 \beta}\left\|\psi_{s}\right\|_{H_{0}^{1}} \\
& \underset{\leq}{\text { Young ineq. }} \frac{\kappa_{0}}{2}\left\|\psi_{s}\right\|_{H_{0}^{1}}^{2}+\frac{C_{0}^{2}}{2 \kappa_{0}}\left|A_{s, l}\right|^{(\beta-2) / \beta} \text {. }
\end{aligned}
$$


Let $\Phi:=(\beta-2) / \beta>(N-2) / N$. We have obtained the estimate

$$
\left\|\psi_{s}\right\|_{H_{0}^{1}}^{2} \leq \widetilde{C}_{0}\left|A_{s, l}\right|^{\Phi}, \quad \widetilde{C}_{0}=\frac{C_{0}^{2}}{4 \kappa_{0}^{2}} .
$$

By now using the Stampacchia estimates (see Section 3.2), we obtain the existence of a real $\Lambda$ independent of $l$ such that $\left|A_{\Lambda, l}\right|=0$. Hence, $\left\|\tilde{v}_{l}\right\|_{L^{\infty}(\Omega)} \leq \Lambda, \Lambda=$ $C\left(\left\|\zeta_{0}\right\|_{L^{\infty}(\partial \Omega)},|\Omega|, N, r\right)\left(C_{0} / \kappa_{0}\right)$.

Proof of Proposition 9. We have the following.

(i) Existence and Estimates. Let $\left(v_{l}\right)_{l \geq 1}$ be the sequence given in Lemma 10. Let also $l \geq 1$ be given, and $\tau_{l}:=1 / h\left(e^{l}\right)$. We assume that $\left\|\gamma_{2}\right\|_{L^{r}} \leq \tau_{l}$. It follows from Lemma 10(ii) that $\left\|v_{l}\right\|_{L^{\infty}} \leq K$, where $K=K\left(\kappa_{0}\right.$, DATA) ( $K$ independent of $\left.l\right)$ is the integer defined by $K=[\Lambda]+1$.

Let now $\tau:=\tau_{K}$, and assume that $\left\|\gamma_{2}\right\|_{L^{r}} \leq \tau$. Then, we have $\left\|v_{K}\right\|_{L^{\infty}} \leq K$. Hence, $T_{K}\left(v_{K}\right)=v_{K}$. On the other hand, $v_{K}$ satisfies $\left(S_{K}\right)$, that is

$$
\begin{gathered}
\rho \mathbf{u} \nabla e^{v_{K}}-\operatorname{div}\left(\left(\nu e^{v_{K}}+\kappa\right) \nabla v_{K}\right)=g\left(x, e^{v_{K}}\right) \quad \text { in } \Omega, \\
v_{K}=\ln \zeta_{0} \quad \text { on } \partial \Omega .
\end{gathered}
$$

Let $\zeta:=e^{v_{K}}$. We have (see Theorem D.1 in the Appendices) $\zeta \in H^{1}(\Omega) \cap L^{\infty}(\Omega)$ and $\nabla \zeta=\zeta \nabla v_{K}$. Consequently, $\zeta$ is a solution of problem $(S)$.

Moreover, $\|\zeta\|_{L^{\infty}(\Omega)} \leq e^{K}=C\left(\kappa_{0}\right.$, DATA $)$.

On the other hand, $v_{K} \geq-K$ almost everywhere implies that $\zeta \geq e^{-K}$ a.e. in $\Omega$. Hence, we obtain (65) by setting $\zeta_{\min }=$ $e^{-K}$ and $\zeta_{\max }=e^{K}$.

(a) The estimation (66) is obtained as follows.

By using the test function $\psi_{s}^{+}$instead of $\psi_{s}$, we obtain

$$
\zeta_{\max } \leq \Lambda_{1}:=e^{C\left(\left\|\gamma_{1}\right\|_{L^{r}} / \kappa_{0}\right)} .
$$

This last estimation is only a first step in order to obtain the majoration for $\zeta_{\max }$ announced in (66).

In fact, let $\widetilde{\kappa}:=v+(\kappa / \zeta)$. We have $0<\nu+\left(\kappa_{0} / \Lambda_{1}\right)=: \widetilde{\kappa}_{0} \leq$ $\widetilde{\kappa} \leq v+\left(\kappa_{0} / \zeta_{\min }\right)<\infty$, and

$$
\begin{gathered}
\rho \mathbf{u} \cdot \nabla \zeta-\operatorname{div}(\widetilde{\kappa} \nabla \zeta)=g^{-}+g^{+} \quad \text { in } \Omega, \\
\zeta=\zeta_{0} \quad \text { on } \partial \Omega
\end{gathered}
$$

we can then consider the decomposition $\zeta=\zeta_{1}+\zeta_{2}$, where $\zeta_{1}$ (resp., $\left.\zeta_{2}\right) \in H^{1}(\Omega) \cap L^{\infty}(\Omega)$ satisfies the following problem $\left(S_{1}\right)$ (resp., $\left.\left(S_{2}\right)\right)$ :

$$
\begin{gathered}
\rho \mathbf{u} \cdot \nabla \zeta_{1}-\operatorname{div}\left(\widetilde{\kappa} \nabla \zeta_{1}\right)=g^{-} \text {in } \Omega \\
\zeta_{1}=\zeta_{0} \quad \text { on } \partial \Omega, \\
\rho \mathbf{u} \cdot \nabla \zeta_{2}-\operatorname{div}\left(\widetilde{\kappa} \nabla \zeta_{2}\right)=g^{+} \text {in } \Omega \\
\zeta_{2}=0 \quad \text { on } \partial \Omega .
\end{gathered}
$$

Note that the second member in the PDE in $\left(S_{1}\right)$ is negative. Hence, by the maximum principle (see $[15, \mathrm{p} .80]$ or $[16, \mathrm{p}$. 191] for a simplified situation), we obtain $\zeta_{1} \leq\left\|\zeta_{0}\right\|_{L^{\infty}(\partial \Omega)}$ a.e. in $\Omega$.
By using next the Stampacchia technique (see again Section 3.2, Remark 6), we major the function $\zeta_{2}$ as follows:

$$
\zeta_{2} \leq C^{\prime} \frac{\left\|g^{+}\right\|_{L^{r}}}{\widetilde{\kappa}_{0}} \underset{\text { by }(84)}{\leq} e^{C\left(\left\|\gamma_{1}\right\|_{L^{r}} / \kappa_{0}\right)} \frac{\left\|g^{+}\right\|_{L^{r}}}{k_{0}}, \quad C=C(\mathrm{DATA}) .
$$

This leads to the majoration (66).

(ii) Regularity Results. (a) If we assume that $\mathbf{u} \in\left(L^{\infty}(\Omega)\right)^{N}$, then $\rho \mathbf{u} \in\left(L^{\infty}(\Omega)\right)^{N}$. Moreover, by using the estimates (65) the diffusion coefficient $v+(\kappa / \zeta)$ is bounded from above and below, and $g(x, \zeta) \in L^{r}(\Omega)$ with $r>3 / 2$ fixed. Hence (see Lemma 5) $g(x, \zeta) \in W^{-1, \beta}(\Omega)$ with $\beta>N$. Consequently, by using the De Giorgi-Nash Theorem (see [19, Th. 8.22] ) we obtain $\zeta \in \mathscr{C}^{0, \alpha}(\bar{\Omega})$, for some $\alpha>0$.

(b) Assume that in addition we have the following:

$$
\begin{gathered}
\partial \Omega, \zeta_{0} \text { are of class } \mathscr{C}^{2, \alpha}, \quad g \in \mathscr{C}^{0, \alpha}\left(\bar{\Omega} \times \mathbb{R}^{+}\right), \\
\rho \mathbf{u} \in\left(\mathscr{C}^{1, \alpha}(\bar{\Omega})\right)^{N}, \quad \kappa \in \mathscr{C}^{1, \alpha}(\bar{\Omega}) .
\end{gathered}
$$

We have proved in the previous point that $\zeta \in \mathscr{C}^{0, \alpha}(\bar{\Omega})$. We now iterate the Schauder estimates as follows. In a first step we see that $g(x, \zeta)$ and $\nu+(\kappa / \zeta)$ are in $\mathscr{C}^{0, \alpha}(\bar{\Omega})$, and by applying Theorem 2.7 in [20] we obtain $\zeta \in \mathscr{C}^{1, \alpha}(\bar{\Omega})$. Consequently, we now obtain (see Appendix B in [11]) $g(x, \zeta), v+(\kappa / \zeta) \epsilon$ $\mathscr{C}^{1, \alpha}(\bar{\Omega})$, and by using Theorem 2.8 in [20] we finally obtain $\zeta \in \mathscr{C}^{2, \alpha}(\bar{\Omega})$. Hence, $\zeta$ is a classical solution of $(S)$.

4.2. Proof of Proposition 7. Let $n \in \mathbb{N}$, and let $\theta_{n}, \varphi_{n}$ be given. We assume that condition $\left(K_{n}\right)$ is satisfied. Recall that this implies in particular $\varphi_{n} \leq \varphi_{\max }$, where $\varphi_{\max }>\|b\|_{L^{\infty}(\partial \Omega)}$ was fixed in (60). Hence, let $\varepsilon:=\varphi_{\max }-\|b\|_{L^{\infty}(\partial \Omega)}>0$.

Step 1. We introduce

$$
\kappa^{(n)}:=\frac{C_{\theta}^{(n)}}{\varphi_{n}}, \quad g_{\theta}(x, u):=C_{5}-C_{3} F u^{2}+C_{4} D u .
$$

Hence, the first subproblem in $\left(Q_{n}\right)$ reads as

$$
\begin{gathered}
\rho \mathbf{u} \cdot \nabla \theta_{n+1}-\operatorname{div}\left(\left(\nu+\frac{\kappa^{(n)}}{\theta_{n+1}}\right) \nabla \theta_{n+1}\right) \\
=g_{\theta}\left(x, \theta_{n+1}\right) \quad \text { in } \Omega \\
\theta_{n+1}=a \quad \text { on } \partial \Omega .
\end{gathered}
$$

Moreover, it is easy to verify that

$$
\begin{gathered}
0<\underbrace{\frac{\alpha_{\theta}}{\varphi_{\max }} \leq \kappa}_{=: \kappa_{0}} \leq\left\|\frac{C_{\theta}^{(n)}}{\varphi_{n}}\right\|_{L^{\infty}}<\infty \\
g_{\theta}^{+}(x, u)=C_{5}+C_{4} D^{+} u, \quad g_{\theta}^{-}(x, u)=-C_{3} F u^{2}+C_{4} D^{-} u .
\end{gathered}
$$


Note also that $\kappa_{0}$ is independent of $n$. Hence, we can apply Proposition 9(i) (take $\zeta=\theta_{n+1}, \kappa=\kappa^{(n)}, g=g_{\theta}, \gamma_{1}=C_{5}$, $\left.\gamma_{2}=C_{4} D^{+}, h(t)=t, \gamma=C_{3} F+C_{4}\left|D^{-}\right|, \zeta_{0}=a\right)$. We obtain the existence of $\tau_{0}>0$ independent of $n$ such that if $\left\|D^{+}\right\|_{L^{r}} \leq$ $\tau_{0}$, then problem $\left(Q_{n} \cdot 1\right)$ admits at least one weak solution $\theta_{n+1} \in H^{1}(\Omega) \cap L^{\infty}(\Omega)$. Moreover, we have

$$
0<e^{-C \varphi_{\max }} \leq \theta_{n+1} \leq e^{C \varphi_{\max }}<\infty, \quad C=C(\text { DATA }) .
$$

Step 2. Let now

$$
\begin{gathered}
\widetilde{\kappa}^{(n)}:=\frac{C_{\varphi}^{(n)}}{\theta_{n+1}}, \\
g_{\varphi}^{(n)}(x, u)=g_{\varphi}^{(n)}(x)=-\varphi_{n}\left(C_{8} \theta_{n+1}^{-1}+C_{6} \theta_{n+1} F-C_{7} D\right) .
\end{gathered}
$$

With these notations, the second subproblem in $\left(Q_{n}\right)$ reads as

$$
\begin{gathered}
\rho \mathbf{u} \cdot \nabla \varphi_{n+1}-\operatorname{div}\left(\left(\nu+\frac{\widetilde{\kappa}^{(n)}}{\varphi_{n+1}}\right) \nabla \varphi_{n+1}\right)=g_{\varphi}^{(n)} \text { in } \Omega, \\
\varphi_{n+1}=b \quad \text { on } \partial \Omega .
\end{gathered}
$$

We verify that

$$
\begin{gathered}
0<\underbrace{\frac{\alpha_{\varphi}}{\theta_{\max }} \leq \widetilde{\kappa}^{(n)} \leq \frac{\left\|C_{\varphi}^{(n)}\right\|_{L^{\infty}}}{\theta_{\min }}<\infty,}_{=: \widetilde{\kappa}_{0}} \\
g_{\varphi}^{+}=\varphi_{n} C_{7} D^{+} \leq \varphi_{\max } C_{7} D^{+}, \\
g_{\varphi}^{-}=-\varphi_{n}\left(C_{8} \theta_{n+1}^{-1}+C_{6} \theta_{n+1} F-C_{7} D^{-}\right), \\
\left|g_{\varphi}^{-}\right|^{\text {by (93) }} \leq \widetilde{C}\left(\text { DATA }, \varphi_{\max }\right) .
\end{gathered}
$$

Hence, we can apply the Proposition 9(i) (take now $\zeta=\varphi_{n+1}$, $\kappa=\widetilde{\kappa}^{(n)}, g=g_{\varphi}^{(n)}, \gamma_{1}=\varphi_{\max } C_{7} D^{+}, h=0, \gamma=\widetilde{C}, \zeta_{0}=$ $b$ ). Then, we obtain the existence of a weak solution (at this stage there is nomore additional condition needed for $\left\|D^{+}\right\|_{L^{r}}$ because $h=0) \varphi_{n+1} \in H^{1}(\Omega) \cap L^{\infty}(\Omega)$ for problem $\left(Q_{n} \cdot 2\right)$. Moreover, we have

$$
0<e^{-C^{\prime} e^{C \varphi_{\max }}} \leq \varphi_{n+1} \leq e^{C^{\prime} e^{C \varphi_{\max }}}<\infty,
$$

where $C, C^{\prime}$ depend on DATA but not on $n$. Moreover, by using (66), we have

$$
\begin{aligned}
\left\|\varphi_{n+1}\right\|_{L^{\infty}(\Omega)} \leq & \|b\|_{L^{\infty}(\partial \Omega)}+C^{\prime \prime}\left\|g_{\varphi}^{+}\right\|_{L^{r}} e^{C \varphi_{\max }}\left\|\varphi_{n+1}\right\|_{L^{\infty}(\Omega)}, \\
\leq & \|b\|_{L^{\infty}(\partial \Omega)}=C^{\prime \prime}(\mathrm{DATA}), \\
& +\underbrace{C^{\prime \prime}\left\|C_{7}\right\|_{L^{\infty}} e^{C \varphi_{\max }}}_{:=K\left(\varphi_{\max }, \text { DATA }\right)}\left\|D^{+}\right\|_{L^{r}}\left\|\varphi_{n+1}\right\|_{L^{\infty}(\Omega)} .
\end{aligned}
$$

Assume now that

$$
\left\|D^{+}\right\|_{L^{r}} \leq \tau:=\min \left(\tau_{0}, \frac{\varepsilon}{\varphi_{\max } K}\right) .
$$

Then, (94) leads to

$$
\left\|\varphi_{n+1}\right\|_{L^{\infty}(\Omega)} \leq\|b\|_{L^{\infty}(\partial \Omega)}+\frac{\varepsilon}{\varphi_{\max }}\left\|\varphi_{n+1}\right\|_{L^{\infty}(\Omega)},
$$

and it follows

$$
\left\|\varphi_{n+1}\right\|_{L^{\infty}(\Omega)} \leq \frac{\|b\|_{L^{\infty}(\partial \Omega)}}{1-\left(\varepsilon / \varphi_{\max }\right)} \leq \varphi_{\max } .
$$

Step 3. If we assume that $\left\|D^{+}\right\|_{L^{r}} \leq \tau$, then by using the results established in the previous two steps, we conclude that there exists a solution $\left(\theta_{n+1}, \varphi_{n+1}\right) \in\left(H^{1} \cap L^{\infty}\right)^{2}$ for problem $\left(Q_{n}\right)$. Moreover, this solution satisfies $\left(K_{n+1}\right)$, and (61) holds.

\section{Proofs of the Theorems}

We begin by a lemma.

Lemma 11. Under the assumptions of Proposition 7, we can extract a subsequence (still denoted by $\left(\theta_{n}, \varphi_{n}\right)$ ) such that

$$
\begin{gathered}
\theta_{n} \stackrel{*}{\rightarrow} \theta, \quad \varphi_{n} \stackrel{*}{\rightarrow} \varphi \quad \text { in } L^{\infty}(\Omega), \\
\theta_{n} \rightarrow \theta, \quad \varphi_{n} \rightarrow \varphi \quad \text { in } H^{1}(\Omega), \\
\frac{1}{\theta_{n} \varphi_{n}} \longrightarrow \frac{1}{\theta \varphi}, \quad C_{\theta}^{(n)} \longrightarrow C_{\theta}(x, \theta, \varphi), \\
C_{\varphi}^{(n)} \longrightarrow C_{\varphi}(x, \theta, \varphi) \quad \text { in } L^{p}(\Omega), p<\infty .
\end{gathered}
$$

Proof. The first properties in (98) follow directly from Proposition 7. By next using $\theta_{n}-\theta_{0}$ as test function in $\left(Q_{n} \cdot 1\right)$ and $\varphi_{n}-\varphi_{0}$ as test function in $\left(Q_{n} \cdot 2\right)$ we obtain a uniform bound for $\left(\theta_{n}\right)$ and $\left(\varphi_{n}\right)$ in the $H^{1}$-norm. Hence, the second properties in (98) follow. Finally, property (99) is obtained by using the dominated convergence theorem. In fact, we have

$$
\begin{gathered}
\frac{1}{\theta_{n} \varphi_{n}} \longrightarrow \frac{1}{\theta \varphi}, \quad C_{\theta}^{(n)} \longrightarrow C_{\theta}(x, \theta, \varphi), \\
C_{\varphi}^{(n)} \longrightarrow C_{\varphi}(x, \theta, \varphi) \quad \text { a.e in } \Omega, \\
\left|\frac{1}{\theta_{n} \varphi_{n}}\right| \leq \frac{1}{\theta_{\min } \varphi_{\min }}, \\
\left|C_{\theta}^{(n)}\right| \leq \sup _{(v, w) \in \mathscr{K}} C_{\theta}(x, v, w), \\
\left|C_{\varphi}^{(n)}\right| \leq \sup _{(v, w) \in \mathscr{K}} C_{\varphi}(x, v, w),
\end{gathered}
$$

where $\mathscr{K}=\left[0, \theta_{\max }\right] \times\left[0, \varphi_{\max }\right]$. 
5.1. Proof of Theorem 1. By using (98) together with (99) we obtain

$$
\begin{gathered}
\rho \mathbf{u} \cdot \nabla \theta_{n+1} \rightarrow \rho \mathbf{u} \cdot \nabla \theta \quad \text { in } L^{1}(\Omega), \\
\left(\nu+\frac{C_{\theta}^{(n)}}{\theta_{n+1} \varphi_{n}}\right) \nabla \theta_{n+1} \rightarrow\left(\nu+\frac{C_{\theta}}{\theta \varphi}\right) \nabla \theta \\
\text { in } L^{q}(\Omega), q<2, \\
\rho \mathbf{u} \cdot \nabla \varphi_{n+1} \rightarrow \rho \mathbf{u} \cdot \nabla \varphi \quad \text { in } L^{1}(\Omega), \\
\left(v+\frac{C_{\varphi}^{(n)}}{\theta_{n+1} \varphi_{n+1}}\right) \nabla \varphi_{n+1} \rightarrow\left(\nu+\frac{C_{\varphi}}{\theta \varphi}\right) \nabla \varphi \\
\operatorname{in} L^{q}(\Omega), q<2 .
\end{gathered}
$$

Moreover by using (98) together with the property $\theta_{n+1} \geq$ $\theta_{\text {min }}>0$, we obtain

$$
\begin{gathered}
\theta_{n+1}^{2} \longrightarrow \theta^{2}, \quad \theta_{n+1} \longrightarrow \theta, \\
\theta_{n+1}^{-1} \longrightarrow \theta^{-1} \quad \text { in } L^{p}(\Omega), p<\infty .
\end{gathered}
$$

Hence we can pass to the limit in the approximate problems $\left(Q_{n}\right)$. We obtain a weak solution $(\theta, \varphi)$ for problem $(Q)$. That is for all $\psi \in \mathscr{D}(\Omega)$ :

$$
\begin{gathered}
\int_{\Omega} \rho \mathbf{u} \cdot \nabla \theta \psi+\int_{\Omega}\left(\nu+\frac{C_{\theta}}{\theta \varphi}\right) \nabla \theta \cdot \nabla \psi \\
=\int_{\Omega}\left(C_{5}-C_{3} F \theta^{2}+C_{4} \theta D\right) \psi, \\
\int_{\Omega} \rho \mathbf{u} \cdot \nabla \varphi \psi+\int_{\Omega}\left(\nu+\frac{C_{\varphi}}{\theta \varphi}\right) \nabla \varphi \cdot \nabla \psi \\
=\int_{\Omega}-\varphi\left(C_{8} \theta^{-1}+C_{6} \theta F-C_{7} D\right) \psi, \\
\theta=a, \quad \varphi=b \quad \text { on } \partial \Omega .
\end{gathered}
$$

Moreover, this solution satisfies

$$
\begin{gathered}
\theta, \varphi \in H^{1}(\Omega) \cap L^{\infty}(\Omega), \\
\theta, \varphi \geq \min \left(\theta_{\min }, \varphi_{\min }\right)>0 \quad \text { a.e. in } \Omega .
\end{gathered}
$$

5.2. Proof of Theorem 2. When $N=2$, the function $F$ has a stronger property of positivity (see Lemma B.1 in the Appendices):

$$
F \geq \frac{D^{2}}{3} .
$$

We will see that this last property allows one to obtain a weak solution for problem $(Q)$ under the assumptions $\left(A_{0}\right)$ and $\left(A_{1}\right)$ but without assuming a low compressibility condition of the form (23).

In order to prove this result, we take over the proof of Proposition 7 with slight modifications: if $\left(\theta_{n}, \varphi_{n}\right)$ is given and satisfies $\left(H_{n}\right)$ (it is not useful to consider $\left(K_{n}\right)$ here), then problem $\left(Q_{n}\right)$ has at least one solution $\left(\theta_{n+1}, \varphi_{n+1}\right)$ satisfying in addition $\left(H_{n+1}\right)$ and the estimates (61).

Step 1. By using property (105) we major $g_{\theta}^{+}$as follows:

$$
\begin{aligned}
g_{\theta}^{+}(x, u) & =C_{5}+C_{4} D^{+} u-C_{3} F u^{2} \\
& \leq C_{5}+C_{4} D^{+} u-\frac{C_{3}}{3}\left(D^{+} u\right)^{2} \\
& \leq C_{5}+\underbrace{D^{+} u}_{\geq 0} \underbrace{\left(C_{4}-\frac{C_{3}}{3}\left(D^{+} u\right)\right)}_{\leq 0 \text { if } D^{+} u \geq\left(3 C_{4} / C_{3}\right)} \leq C_{5}+\frac{3 C_{4}^{2}}{C_{3}} .
\end{aligned}
$$

Hence, we have here estimated $g_{\theta}^{+}$independently of the second variable. We then apply Proposition 9(i), but now we take $\gamma_{1}=C_{5}+\left(3 C_{4}^{2} / C_{3}\right)$ instead of $C_{5}$ and $h(t)=0$ instead of $h(t)=t$. It follows that there exists (without any condition on $\left\|D^{+}\right\|_{L^{r}}$ because $h=0$ ) a weak solution $\theta_{n+1} \in H^{1}(\Omega) \cap L^{\infty}(\Omega)$ for problem $\left(Q_{n} \cdot 1\right)$, with the estimate

$$
0<e^{-C\left\|\varphi_{n}\right\|_{L^{\infty}}} \leq \theta_{n+1} \leq e^{C\left\|\varphi_{n}\right\|_{L^{\infty}}}<\infty, \quad C=C(\text { DATA }) .
$$

Step 2. By taking over the arguments presented in the proof of Proposition 7 we see that problem $\left(Q_{n} \cdot 2\right)$ has at least one positive solution $\varphi_{n+1} \in H^{1}(\Omega) \cap L^{\infty}(\Omega)$.

Hence, at this point we have obtained a weak solution $\left(\theta_{n+1}, \varphi_{n+1}\right)$ for $\left(Q_{n}\right)$ satisfying in addition $\left(H_{n+1}\right)$. It remains to prove that the estimates (61) hold. We have made a first step in this direction by proving (107). We will now prove

$$
0<e^{-C\left\|\theta_{n+1}\right\|_{L^{\infty}}} \leq \varphi_{n+1} \leq\|b\|_{L^{\infty}(\partial \Omega)}, \quad C=C(\text { DATA }) .
$$

In fact, by using the additional assumption $\left(A_{1}\right)$ we major the function $g_{\varphi}^{(n)}$ as follows:

$$
\begin{aligned}
& g_{\varphi}^{(n)}(x)=-\varphi_{n}\left(C_{8} \theta_{n+1}^{-1}+C_{6} \theta_{n+1} F-C_{7} D\right) \\
& \stackrel{\text { by }(106)}{\leq}-\varphi_{n}\left(C_{8} \theta_{n+1}^{-1}+\frac{C_{6}}{3} \theta_{n+1} D^{2}-C_{7} D\right) \\
& \quad \leq-\frac{\rho \varphi_{n}}{\theta_{n+1}}\left(c_{8}+\frac{c_{6}}{3}\left(\theta_{n+1} D\right)^{2}-c_{7}\left(\theta_{n+1} D\right)\right) \\
& \quad=-\frac{\rho \varphi_{n}}{\theta_{n+1}} \mathscr{P}\left(\theta_{n+1} D\right),
\end{aligned}
$$

with $\mathscr{P}(X):=\left(c_{6} / 3\right) X^{2}-c_{7} X+c_{8}$. We remark that the discriminant $\Delta$ of $\mathscr{P}$ is negative: $\Delta=c_{7}^{2}-(4 / 3) c_{6} c_{8}=-4.864 *$ $10^{-3}<0$. It follows that $\mathscr{P}$ is positive and consequently $g_{\varphi}^{(n)}$ is negative. Hence, by applying Proposition 9(i) with now $g^{+} \equiv 0$ we obtain (108).

Step 3. By using (107) together with (108) we obtain the estimates (61). Hence, we have recovered the conclusions of Proposition 7. The remainder of the proof for Theorem 3 is 
exactly the same as for Theorem 1: we can extract a subsequence with the properties (98)-(99). These properties are sufficient to pass to the limit $n \rightarrow \infty$ in $\left(Q_{n}\right)$, and Theorem 2 follows.

5.3. Proof of Theorem 3. Let $(\theta, \varphi)$ be a weak solution for $(Q)$ in the class $\mathcal{S}$, and consider the notations

$$
\begin{gathered}
g_{\theta}(x, u):=C_{5}-C_{3} F u^{2}+C_{4} D u, \\
g_{\varphi}(x, u):=-u\left(C_{8} \theta^{-1}+C_{6} \theta F-C_{7} D\right) .
\end{gathered}
$$

(i) It suffices to remark that the coefficients $C_{\theta} / \varphi$ and $C_{\varphi} / \theta$ are bounded from above and below, and $g_{\theta}, g_{\varphi}$ are Caratheodory functions satisfying (63) and (64). Hence, we can apply the first point in Proposition 9(ii) in each equation of $(Q)$. We obtain $\theta, \varphi \in \mathscr{C}^{0, \alpha}(\bar{\Omega})$, for some $\alpha>0$.

(ii) Assume that in addition we have the following:

$\partial \Omega, a, b$ are of class $\mathscr{C}^{2, \alpha}, \quad F \in \mathscr{C}^{0, \alpha}(\bar{\Omega})$,

$$
\rho \mathbf{u} \in\left(\mathscr{C}^{1, \alpha}(\bar{\Omega})\right)^{N}, \quad C_{\theta}, C_{\varphi} \in \mathscr{C}^{1, \alpha}\left(\bar{\Omega} \times\left(\mathbb{R}^{+}\right)^{2}\right) .
$$

We remark now that the conditions in the second part of Proposition 9(ii) are satisfied for each equation of $(Q)$. Hence, $\theta, \varphi \in \mathscr{C}^{2, \alpha}(\bar{\Omega})$, and it is a classical solution of $(Q)$.

\section{Appendices}

\section{A. Derivation of the $\theta-\varphi$ Model}

The model is constructed from the $k-\varepsilon$ one which takes the form

$$
\begin{aligned}
\partial_{t} k & +\mathbf{u} \cdot \nabla k-\frac{c_{v}}{\rho} \operatorname{div}\left(\left(\nu+\rho \frac{k^{2}}{\varepsilon}\right) \nabla k\right) \\
& =c_{v} \frac{k^{2}}{\varepsilon} F-\frac{2}{3} k D-\varepsilon \\
\partial_{t} \varepsilon & +\mathbf{u} \cdot \nabla \varepsilon-\frac{c_{\varepsilon}}{\rho} \operatorname{div}\left(\left(\nu+\rho \frac{k^{2}}{\varepsilon}\right) \nabla \varepsilon\right) \\
& =c_{1} k F-\frac{2 c_{1}}{3 c_{v}} \varepsilon D-c_{2} \frac{\varepsilon^{2}}{k},
\end{aligned}
$$

where $D(x, t):=\operatorname{div} \mathbf{u}(x, t), F(x, t):=(1 / 2)\left|\nabla \mathbf{u}+(\nabla \mathbf{u})^{T}\right|^{2}-$ $(2 / 3) D(x, t)^{2} \geq 0$ (see Appendix B) and $c_{v}, c_{\varepsilon}, c_{1}, c_{2}$ are generally taken as positive constants. Their usual values are (see [1, page 122])

$$
c_{v}=0.09, \quad c_{\varepsilon}=0.07, \quad c_{1}=0.128, \quad c_{2}=1.92 .
$$

We then consider the new variables

$$
\theta=\frac{k}{\varepsilon}, \quad \varphi=k^{\alpha} \varepsilon^{\beta},
$$

with $\alpha$ and $\beta$ to be chosen appriopriately. Let $D_{t}$ denote the total derivative operator. By using (A.1) together with (A.2) we obtain an equation for $\theta$ :

$$
\begin{gathered}
D_{t} \theta=\frac{\partial \theta}{\partial t}+\mathbf{u} \cdot \nabla \theta=\frac{1}{\varepsilon} D_{t} k-\frac{k}{\varepsilon^{2}} D_{t} \varepsilon \\
=-c_{3} \theta^{2} F+c_{4} \theta D+c_{5}+\mathrm{Diff}_{\theta}, \\
c_{3}=c_{1}-c_{v}, \quad c_{4}=\frac{2}{3}\left(\frac{c_{1}}{c_{v}}-1\right), \quad c_{5}=c_{2}-1,
\end{gathered}
$$

where $\operatorname{Diff}_{\theta}$ denotes the collected terms coming from the viscous one in the $k$ and $\varepsilon$ equations. The equation for $\varphi$ is obtained in the same way:

$$
\begin{aligned}
D_{t} \varphi= & \alpha k^{\alpha-1} \varepsilon^{\beta} D_{t} k+\beta k^{\alpha} \varepsilon^{\beta-1} D_{t} \varepsilon \\
= & \alpha k^{\alpha-1} \varepsilon^{\beta}\left(c_{v} \frac{k^{2}}{\varepsilon} F-\frac{2}{3} k D-\varepsilon\right)+\operatorname{Diff} 1_{\varphi} \\
& +\beta k^{\alpha} \varepsilon^{\beta-1}\left(c_{1} k F-\frac{2 c_{1}}{3 c_{v}} \varepsilon D-c_{2} \frac{\varepsilon^{2}}{k}\right)+\operatorname{Diff} 2_{\varphi} \\
= & F k^{\alpha+1} \varepsilon^{\beta-1}\left(\alpha c_{v}+\beta c_{1}\right) \\
& -k^{\alpha} \varepsilon^{\beta} D \frac{2}{3}\left(\alpha+\beta \frac{c_{1}}{c_{v}}\right)-k^{\alpha-1} \varepsilon^{\beta+1}\left(\alpha+\beta c_{2}\right)+\operatorname{Diff}{ }_{\varphi}, \\
= & -\varphi\left(c_{6} \theta F-c_{7} D+c_{8} \theta^{-1}\right)+\operatorname{Diff}, \\
c_{6}, \quad & \alpha c_{v}-\beta c_{1}, \quad c_{7}=-\frac{2}{3}\left(\alpha+\beta \frac{c_{1}}{c_{v}}\right), \quad c_{8}=\alpha+\beta c_{2},
\end{aligned}
$$

where $\operatorname{Diff}_{\varphi}=\operatorname{Diff} 1_{\varphi}+\operatorname{Diff} 2_{\varphi}$ is the sum of the terms coming from the viscous one in the $k$ and $\varepsilon$ equations.

The usual constant values for the parameters $c_{3}, c_{4}$, and $c_{5}$ appearing in the model are obtained by replacing the values (A.3) in the expressions (A.5). This leads to

$$
c_{3}=0.038, \quad c_{4}=0.2815, \quad c_{5}=0.92 .
$$

At this stage it remains to choose appropriately $\alpha$ and $\beta$ in (A.4) and to model the terms $\operatorname{Diff}_{\theta}$ and $\operatorname{Diff}_{\varphi}$.

It is shown in [1, page 67], that a good choice in the incompressible situation (i.e., when $D=0$ ) is for instance $\alpha=-3, \beta=2$. This leads to the following constant values:

$$
c_{6}=0.014, \quad c_{7}=0.104, \quad c_{8}=0.84,
$$

and this makes the dynamic stable for the equation in $\varphi$. That is, in the absence of the viscous part $\operatorname{Diff}_{\varphi}$, we have $D_{t} \varphi \leq 0$.

In the compressible situation the authors suggest in $[1$, page 125], to consider another choice: $\alpha=-2\left(c_{1} / c_{\nu}\right) \approx-2.88$ and $\beta=2$ which makes again the dynamic stable.

Nevertheless, in this last situation, the variable $\varphi$ does not have a clear physical meaning (whereas when $\alpha=-3, \beta=2$, we have $\varphi=\varepsilon^{2} / k^{3}$, and $L:=\varphi^{-1 / 2}$ represents a length scale of turbulence (see [2])). Moreover, a careful estimation shows 
(see Lemma B.1 in Appendix B) that when $N=2$, we have $F \geq(1 / 3) D^{2}$. This leads to

$$
\begin{aligned}
- & \varphi\left(c_{6} \theta F-c_{7} D+c_{8} \theta^{-1}\right) \\
\leq- & \frac{\varphi}{\theta}\left(\frac{c_{6}}{3}(\theta D)^{2}-c_{7}(\theta D)+c_{8}\right)=-\frac{\varphi}{\theta} \mathscr{P}(\theta D),
\end{aligned}
$$

with $\mathscr{P}(X):=\left(c_{6} / 3\right) X^{2}-c_{7} X+c_{8}$. Hence, the choice $\alpha=-3$, $\beta=2$ makes again the dynamic stable when $N=2$. In fact, in this case $c_{6}, c_{7}, c_{8}$ take the values (A.8), and the discriminant $\Delta$ of $\mathscr{P}$ is $\Delta=c_{7}^{2}-(4 / 3) c_{6} c_{8}=-4.864 * 10^{-3}<0$. Consequently, $\mathscr{P}(X) \geq 0$ and $D_{t} \varphi \leq 0$ in the absence of the viscous terms.

In consequence we point out that the choice $\alpha=-3$, $\beta=2$ is also interesting in the compressible situation. We shall make this choice in all the situations. Our analysis (see Theorem 1) shows that this leads to a well-posed model even when $N=3$ under an additional assumption of low compressibility of the flow.

The terms $\operatorname{Diff}_{\theta}$ and $\operatorname{Diff}_{\varphi}$ are modelled (see [7]) by

$$
\begin{aligned}
\operatorname{Diff}_{\theta} & =\frac{1}{\rho} \operatorname{div}\left(\left(v+c_{\theta} v_{t}\right) \nabla \theta\right), \\
\operatorname{Diff}_{\varphi} & =\frac{1}{\rho} \operatorname{div}\left(\left(v+c_{\varphi} v_{t}\right) \nabla \theta\right),
\end{aligned}
$$

where $v_{t}:=\rho c_{\nu}\left(k^{2} / \varepsilon\right)=\rho\left(c_{v} / \theta \varphi\right)$ is the turbulent viscosity coming from the equation of $k$, and $c_{\theta}, c_{\varphi}$ are two new parameters for the model.

The determination of the parameters $c_{\theta}$ and $c_{\varphi}$ can be realized in the same way as for the determination of the coefficients arising in the $k-\varepsilon$ model (see [1]). In [9] a constant value for both $c_{\theta}$ and $c_{\varphi}$ was numerically tuned from a simulation of a Poiseuil flow. However, better results are obtained if we allow $c_{\theta}, c_{\varphi}$ to be some positive functions (see [5]). In our analysis we allow the coefficients to be of a very general form; in particular, $c_{\theta}, c_{\varphi}$ may depend on $x, \theta$, and $\varphi$. We only assume that they are Caratheodory functions and that they satisfy some positivity and boundedness properties (see (18)-(20), where $C_{\theta}=\rho c_{\theta}$ and $C_{\varphi}=\rho c_{\varphi}$ ).

\section{B. Positivity of the Function $F$}

In this paragraph we will establish some properties of positivity for the function $F$ appearing in the models.

Let $\mathscr{M}_{N}(\mathbb{R})$ denote the vector space of the $N$-square matrix with real coefficients, equipped with the scalar product:

$$
A: B=\sum_{i} \sum_{j} a_{i j} b_{i j}, \quad \forall A=\left(a_{i j}\right), B=\left(b_{i j}\right) \in \mathscr{M}_{N}(\mathbb{R}) \text {. }
$$

Hence, $|A|^{2}:=\sqrt{A: A}$ defines a norm on $\mathscr{M}_{N}(\mathbb{R})$.
For a vector field $\mathbf{u}: \Omega \rightarrow \mathbb{R}^{N}$ we classically define the gradient $\nabla \mathbf{u}$ and the divergence $\operatorname{div} \mathbf{u}(=: D)$ by

$$
\begin{gathered}
\nabla \mathbf{u}: \Omega \longrightarrow \mathscr{M}_{N}(\mathbb{R}), \quad(\nabla \mathbf{u}(x))_{i j}=\frac{\partial u_{i}(x)}{\partial x_{j}}, \\
D: \Omega \longrightarrow \mathbb{R}, \quad D(x)=\sum_{i} \frac{\partial u_{i}(x)}{\partial x_{i}}=\operatorname{Tr}(\nabla \mathbf{u})
\end{gathered}
$$

Recall that the function $F$ was defined by the formula

$$
F(x):=\frac{1}{2}\left|\nabla \mathbf{u}+(\nabla \mathbf{u})^{T}\right|^{2}-\frac{2}{3} D(x)^{2},
$$

and by an easy calculation, we obtain

$$
F(x)=\left(\nabla \mathbf{u}+(\nabla \mathbf{u})^{t}\right): \nabla \mathbf{u}-\frac{2}{3} D(x)^{2} .
$$

This last expression is sometimes chosen (for instance in [1]) to equivalently define $F$.

The important fact is that we always have $F \geq 0$ but moreover, when $N=2$, the stronger estimate $F \geq(1 / 3) D^{2}$ holds. These properties are established in the following lemma.

Lemma B.1. The function $F$ satisfies the estimates

$$
\begin{aligned}
F= & \frac{2}{3}\left(\left(\partial_{1} u_{1}-\partial_{2} u_{2}\right)^{2}+\left(\partial_{1} u_{1}-\partial_{3} u_{3}\right)^{2}+\left(\partial_{2} u_{2}-\partial_{3} u_{3}\right)^{2}\right) \\
& +\left(\partial_{2} u_{1}+\partial_{1} u_{2}\right)^{2}+\left(\partial_{3} u_{1}+\partial_{1} u_{3}\right)^{2} \\
& +\left(\partial_{3} u_{2}+\partial_{2} u_{3}\right)^{2} \geq 0, \quad \text { when } N=3 \\
F= & \left(\partial_{1} u_{1}-\partial_{2} u_{2}\right)^{2}+\left(\partial_{2} u_{1}+\partial_{1} u_{2}\right)^{2} \\
+ & \frac{1}{3} D^{2} \geq \frac{1}{3} D^{2}, \quad \text { when } N=2 .
\end{aligned}
$$

Proof. Let $N=2$ or 3 , and $M:=\nabla \mathbf{u}+(\nabla \mathbf{u})^{t}$. Then $M_{i j}=$ $\partial_{j} u_{i}+\partial_{i} u_{j}$, and we obtain

$$
\begin{aligned}
|M|^{2} & =\sum_{i}\left(\left(2 \partial_{i} u_{i}\right)^{2}+\sum_{j \neq i}\left(\partial_{j} u_{i}+\partial_{i} u_{j}\right)^{2}\right) \\
& =4 \sum_{i}\left(\partial_{i} u_{i}\right)^{2}+2 \sum_{i} \sum_{j>i}\left(\partial_{j} u_{i}+\partial_{i} u_{j}\right)^{2}, \\
F & =\frac{1}{2}|M|^{2}-\frac{2}{3} D^{2} \\
& =\underbrace{2 \sum_{i}\left(\partial_{i} u_{i}\right)^{2}-\frac{2}{3}\left(\sum_{i} \partial_{i} u_{i}\right)^{2}}_{:=A}+\sum_{i} \sum_{j>i}\left(\partial_{j} u_{i}+\partial_{i} u_{j}\right)^{2} .
\end{aligned}
$$

The term $A$ is evaluated separately in the cases $N=2$ and $N=3$. 
We remark that $2\left(a^{2}+b^{2}+c^{2}\right)-(2 / 3)(a+b+c)^{2}=(2 / 3)((a-$ $\left.b)^{2}+(a-c)^{2}+(b-c)^{2}\right)$. Hence, when $N=3$, we have

$$
A=\frac{2}{3}\left(\left(\partial_{1} u_{1}-\partial_{2} u_{2}\right)^{2}+\left(\partial_{1} u_{1}-\partial_{3} u_{3}\right)^{2}+\left(\partial_{2} u_{2}-\partial_{3} u_{3}\right)^{2}\right),
$$

and we obtain the expression announced for $F$.

In the same way, we remark that

$$
\begin{aligned}
2\left(a^{2}+b^{2}\right)-\frac{2}{3}(a+b)^{2}= & \frac{2}{3}(a-b)^{2}+\frac{2}{3}\left(a^{2}+b^{2}\right) \\
= & \frac{2}{3}(a-b)^{2}+\frac{1}{3}(a+b)^{2} \\
& +\frac{1}{3}(a-b)^{2} \\
= & (a-b)^{2}+\frac{1}{3}(a+b)^{2} .
\end{aligned}
$$

Hence, when $N=2$, we obtain

$$
A=\left(\partial_{1} u_{1}-\partial_{2} u_{2}\right)^{2}+\frac{1}{3} \underbrace{\left(\partial_{1} u_{1}+\partial_{2} u_{2}\right)^{2}}_{=D^{2}},
$$

and the expression for $F$ follows.

\section{On the Low Compressibility Assumption}

We will show here that, without any assumption of low compressibility of the form (23), problem (Q) may be very hard to analyze when $N=3$, and singular solutions or nonexistence of weak solution may occur.

When the dimension equals two, we have seen in Theorem 2 that a condition of low compressibility is not necessary. The reason is related to the fact that a stronger property of positivity for $F$ holds in this case; that is, we have $F \geq D^{2} / 3$. When the dimension equals 3 such a property does not hold in general.

In fact, let for instance $\Omega=B_{\mathbb{R}^{3}}(0,1)$ and

$$
\begin{gathered}
\rho=\left(\prod_{i=1}^{3}\left(x_{i}+4\right)\right)^{-1}, \quad \mathbf{u}=\left(u_{1}, u_{2}, u_{3}\right)^{t}, \\
u_{i}=x_{i}+4 .
\end{gathered}
$$

Then, a simple calculation gives $D=3$ and $F=0$. Hence (Q) reads as

$$
\begin{gathered}
\rho \mathbf{u} \cdot \nabla \theta-\operatorname{div}\left(\left(\nu+\frac{C_{\theta}}{\theta \varphi}\right) \nabla \theta\right)=3 C_{4} \theta+C_{5} \quad \text { in } \Omega, \\
\rho \mathbf{u} \cdot \nabla \varphi-\operatorname{div}\left(\left(\nu+\frac{C_{\varphi}}{\theta \varphi}\right) \nabla \varphi\right)=\varphi\left(3 C_{7}-C_{8} \theta^{-1}\right) \quad \text { in } \Omega, \\
\theta=a, \quad \varphi=b \quad \text { on } \partial \Omega .
\end{gathered}
$$

In this situation the problem becomes hard to analyze. Assume, however, that we have obtained a solution $(\theta, \varphi)$ in the class $\mathcal{S}$. Then, the equation satisfied by $\theta$ is closely related to

$$
\begin{gathered}
-\operatorname{div}(\eta \nabla \theta)=3 C_{4} e^{\theta}+C_{5} \quad \text { in } \Omega, \\
\theta=a \quad \text { on } \partial \Omega,
\end{gathered}
$$

with $\eta$ bounded from above and below. Hence, a contradiction can occur because the problem $(R)$ may not have any weak solution (see for instance $[21,22]$ ). Note that in the considered example $\rho$ and $\mathbf{u}$ satsify all the conditions needed in $\left(A_{0}\right)$, except $\mathbf{u} \cdot \mathbf{n}=0$ on $\partial \Omega$, but this is not restrictive for the purpose here. In fact, we can consider the domain $\Omega_{1}=B_{\mathbb{R}^{3}}(0,2)$ which contains $\Omega$, and we can extend $\rho$, $\mathbf{u}$ in $\Omega_{1}$ in such a way that all the conditions in $\left(A_{0}\right)$ are satisfied. Hence, we obtain an example within the main situation of the study, but the evocated problems remain the same.

\section{A Generalized Chain Rule}

Let $u \in W^{1, p}(\Omega)$, and let $G: \mathbb{R} \rightarrow \mathbb{R}$ be a Lipschitz function. We recall here some useful properties of the composed function $G(u)$. In particular, we schall see that $G(u) \in W^{1, p}(\Omega)$. Moreover, in some situations, we also have $\left(\partial / \partial x_{i}\right) G(u)=G^{\prime}(u)\left(\partial u / \partial x_{i}\right)$.

The main result we have in mind is Theorem D.1 which is due to Stampacchia. In particular, we point out that the additional assumption $G(0)=0$ for the Lipschitz function $G$ is only necessary if $\Omega$ is unbounded and $p \neq \infty$ or if we want $G(u)$ to have a vanishing trace on $\partial \Omega$ when $u$ has it (this last situation was in fact the case of interest of Stampacchia).

Theorem D.1. Let $G$ be a Lipschitz real function; let $\Omega \subset \mathbb{R}^{N}$ be a bounded open Lipschitz domain, and $u \in W^{1, p}(\Omega)$, with $p \in[1 ; \infty]$. We have the following:

(i) $G(u) \in W^{1, p}(\Omega)$. Moreover if $u \in W_{0}^{1, p}(\Omega)$ and $G(0)=$ 0 , then $G(u) \in W_{0}^{1, p}(\Omega)$.

(ii) If $G^{\prime}$ has a finite number of discontinuity (the derivative $G^{\prime}$ of $G$ takes here the classical sense). Then, the weak derivatives of $G(u)$ are given by the formula

$$
\frac{\partial}{\partial x_{i}} G(u)=G^{\prime}(u) \frac{\partial u}{\partial x_{i}} \quad \text { a.e. in } \Omega .
$$

Proof. See the appendix in [15] for the original proof or [19, Theorem 7.8] and [6, Theorem 4] for alternative proofs and additional comments.

We also recall that the formula (D.1) may be interpreted in some critical points. In fact, let $\left(t_{i}\right)_{i=1, . ., n}$ denote the points of discontinuity of $G^{\prime}$, and let $E_{i}:=\left\{x \in \Omega: u(x)=t_{i}\right\}$ be the associated level sets for the function $u$. Let $1 \leq i \leq n$ be a fixed integer. If $\left|E_{i}\right|>0$, then the formula (D.1) has a priori no sense in this last set which is not negligible. Nevertheless, it can be shown (see [15]) that $\partial u / \partial x_{i}=0$ on such a set. Hence, we interpret the right hand side of (D.1) as zero in the critical set $E_{i}$. 
We now establish some technical results used in the proof of Lemma 10. Let $v_{l} \in H^{1}(\Omega)$ be given, and consider the function $h_{ \pm} \in L_{\text {loc }}^{1}(\mathbb{R})$ defined by

$$
h_{ \pm}(y):=e^{y}\left(y-T_{s, \pm}(y)\right) .
$$

We introduce the functions

$$
\begin{aligned}
& g_{ \pm}(t):=\int_{0}^{T_{l}(t)} h_{ \pm}(y) d y, \quad t \in \mathbb{R}, \\
& \zeta_{l, \pm}(x):=g_{ \pm}\left(v_{l}(x)\right) \quad \text { a.e. } x \in \Omega .
\end{aligned}
$$

We have the following.

Lemma D.2. The function $\zeta_{l, \pm}$ has the properties

$$
\begin{gathered}
\zeta_{l, \pm} \in H^{1}(\Omega) \cap L^{\infty}(\Omega), \\
\nabla \zeta_{l, \pm}=\nabla e^{T_{l}\left(v_{l}\right)} \psi_{s}^{ \pm} .
\end{gathered}
$$

Proof. A simple majoration gives

$$
\left|\zeta_{l, \pm}(x)\right| \leq \int_{0}^{l}\left|h_{ \pm}(y)\right| d y, \quad \text { a.e. in } \Omega ;
$$

hence, $\zeta_{l, \pm} \in L^{\infty}(\Omega)$.

We next remark that $g_{ \pm}$is a Lipschitz function and its classical derivative is given by

$$
g_{ \pm}^{\prime}(t)=h_{ \pm}\left(T_{l}(t)\right) \mathbf{1}_{\{|t| \leq l\}} \quad \forall t \neq l,-l
$$

Hence, by using Theorem D.1, we obtain $\zeta_{l, \pm} \in H^{1}(\Omega)$ and

$$
\begin{aligned}
\nabla \zeta_{l, \pm} & =h_{ \pm}\left(T_{l}\left(v_{l}\right)\right) \mathbf{1}_{\left\{\left|v_{l}\right| \leq l\right\}} \nabla v_{l}=h_{ \pm}\left(v_{l}\right) \mathbf{1}_{\left\{\left|v_{l}\right| \leq l\right\}} \nabla v_{l} \\
& =e^{v_{l}} \nabla v_{l} \mathbf{1}_{\left\{\left|v_{l}\right| \leq l\right\}} \psi_{s}^{ \pm}\left(v_{l}\right)=\nabla e^{T_{l}\left(v_{l}\right)} \psi_{s}^{ \pm} .
\end{aligned}
$$

\section{References}

[1] B. Mohammadi and O. Pironneau, Analysis of the K-Epsilon Turbulence Model, Masson, Saint-Jean-de-Monts, France, 1994.

[2] D. C. Wilcox, Turbulence Modeling for CFD, chapter 4, DCW Industries, 2nd edition, 1998.

[3] P. L. Lions, Mathematical Topics in Fluid Mechanics, vol. 1, Oxford Science Publications, 1996.

[4] A. N. Kolmogorov, "Energy dissipation in locally isotropic," Doklady Akademii Nauk SSSR, vol. 32, pp. 19-21, 1941.

[5] B. Mohammadi and G. Puigt, "Mathematical and numerical analysis of an alternative well-posed two-layer turbulence model," Mathematical Modelling and Numerical Analysis, vol. 35, no. 6, pp. 1111-1136, 2001.

[6] L. C. Evans and R. F. Gariepy, Measure Theory and Fine Properties of Functions, CRC Press, London, UK, 1992.

[7] R. Lewandowski and B. Mohammadi, "Existence and positivity results for the $\theta, \phi$ and a modified $k-\varepsilon$ two equations turbulence models," Mathematical Methods in the Applied Sciences, vol. 3, no. 2, pp. 195-215, 1993.
[8] M. C. Marmol and F. O. Gallego, "Coupling the stokes and navier-stokes equations with two scalar nonlinear parabolic equations," Mathematical Modelling and Numerical Analysis, vol. 33, no. 1, pp. 157-167, 1999.

[9] B. Mohammadi and J. H. Saiac, "Turbulent compressible axisymetric flows computations with the $k-\varepsilon$ model," International Journal of Computational Fluid Dynamics, vol. 1, pp. 115-133, 1993.

[10] M. C. Mármol and F. O. Gallego, "Existence of solution to nonlinear elliptic systems arising in turbulence modelling," Mathematical Models and Methods in Applied Sciences, vol. 10, no. 2, pp. 247-260, 2000.

[11] P. Dreyfuss, "Results for a turbulent system with unbounded viscosities: weak formulations, existence of solutions, boundedness and smoothness," Nonlinear Analysis: Theory, Methods \& Applications, vol. 68, no. 6, pp. 1462-1478, 2008.

[12] O. Lafitte, "Existence and positivity of a system $k-\varepsilon$ with a production term of the Rayleigh-Taylor type," Applied Mathematics Letters, vol. 15, no. 3, pp. 347-354, 2002.

[13] Z.-N. Wu and F. Fu, "Positivity of $k$-epsilon turbulence models for incompressible flow," Mathematical Models and Methods in Applied Sciences, vol. 12, no. 3, pp. 393-406, 2002.

[14] O. A. Ladyzhenskaya and N. N. Ural'Tseva, Linear and Quasilinear Elliptic Equations, Academic Press, New York, NY, USA, 1968.

[15] G. Stampacchia, Equations Elliptiques du Second Ordre à Coefficients Discontinus, Les Presses de l'Université de Montreal, 1966.

[16] H. Brezis, Analyse Fonctionnelle, Théorie et Applications, Masson, Saint-Jean-de-Monts, France, 1993.

[17] E. Gagliardo, "Carratterizzazioni delle tracce sulla frontiera relative ad alcune classi di funzioni in n variabili," Rendiconti del Seminario Matematico della Universita di Padova, vol. 27, pp. 284-305, 1957.

[18] F. Augsburger and N. Hungerbühler, "Quasilinear elliptic systems in divergence form with weak monotonicity and nonlinear physical data," Electronic Journal of Differential Equations, vol. 2004, no. 144, pp. 1-18, 2004.

[19] D. Gilbarg and N. S. Trudinger, Elliptic Partial Differential Equations of Second Order, Springer, New York, NY, USA, 2nd edition, 1998.

[20] Y.-Z. Chen and L.-C. Wu, Second Order Elliptic Equations and Elliptic Systems, vol. 174 of Translations of Mathematical Monographs, American Mathematical Society, Providence, RI, USA, 1998.

[21] H. Brezis and L. Nirenberg, "Positive solutions of nonlinear elliptic equations involving critical sobolev exponents," Communications on Pure and Applied Mathematics, vol. 36, no. 4, pp. 437-477, 1983.

[22] D. Lupo and K. R. Payne, "Critical exponents for semilinear equations of mixed elliptic-hyperbolic and degenerate types," Communications on Pure and Applied Mathematics, vol. 56, no. 3, pp. 403-424, 2003. 


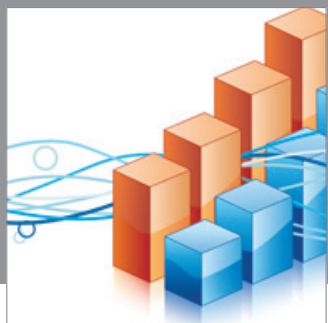

Advances in

Operations Research

mansans

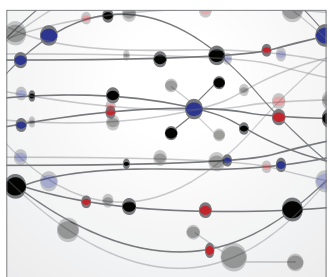

The Scientific World Journal
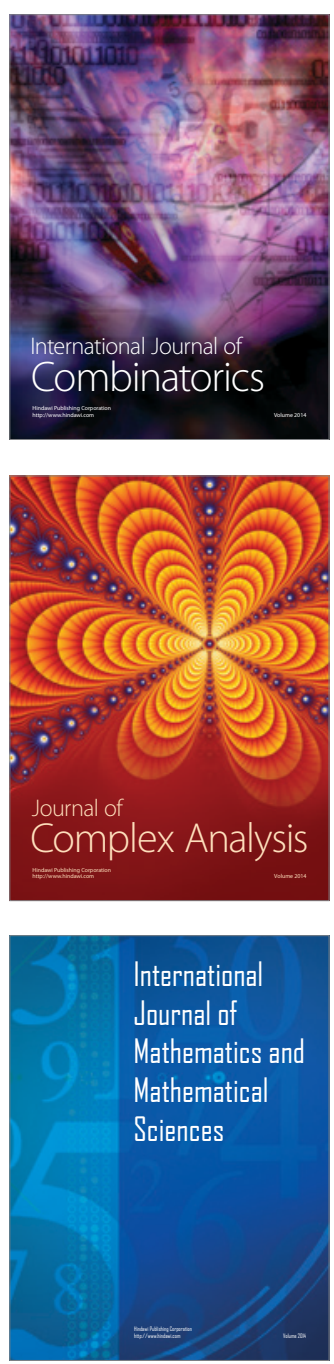
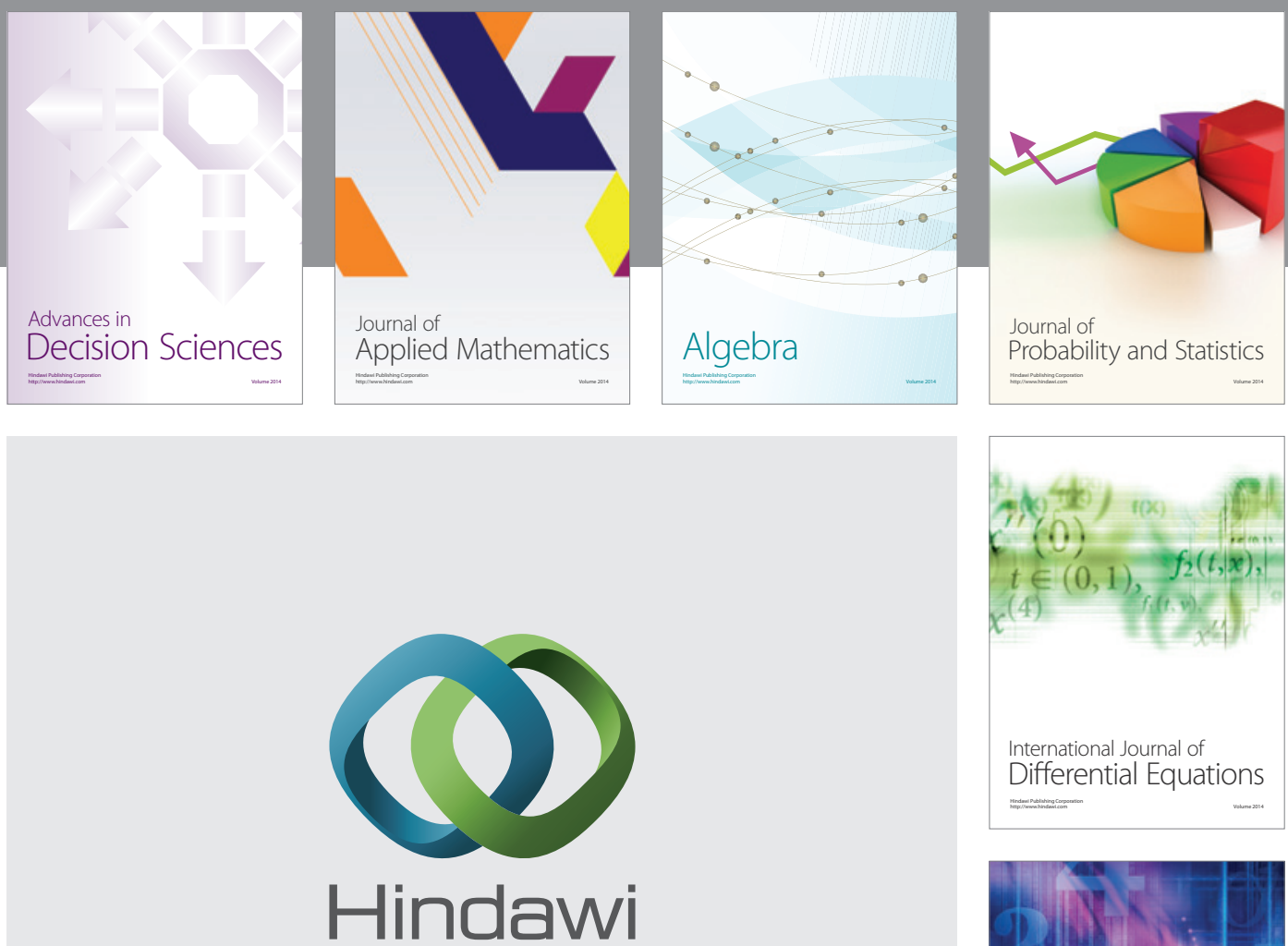

Submit your manuscripts at http://www.hindawi.com
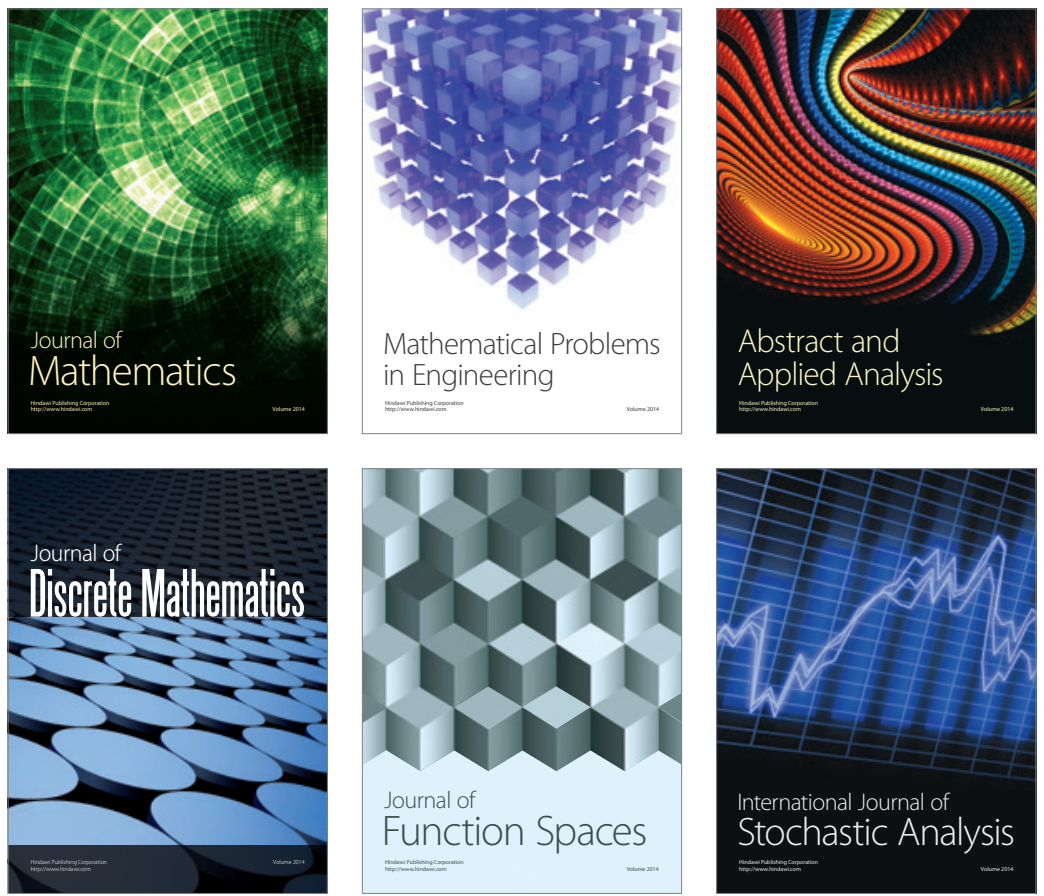

Journal of

Function Spaces

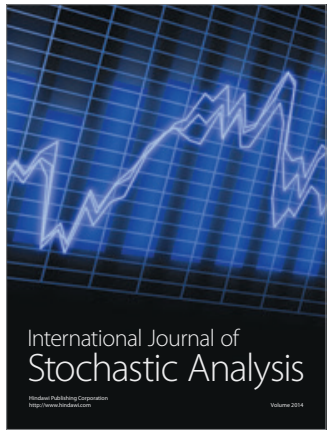

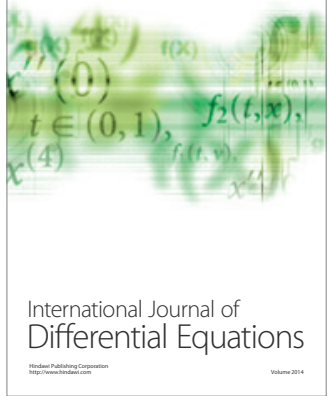
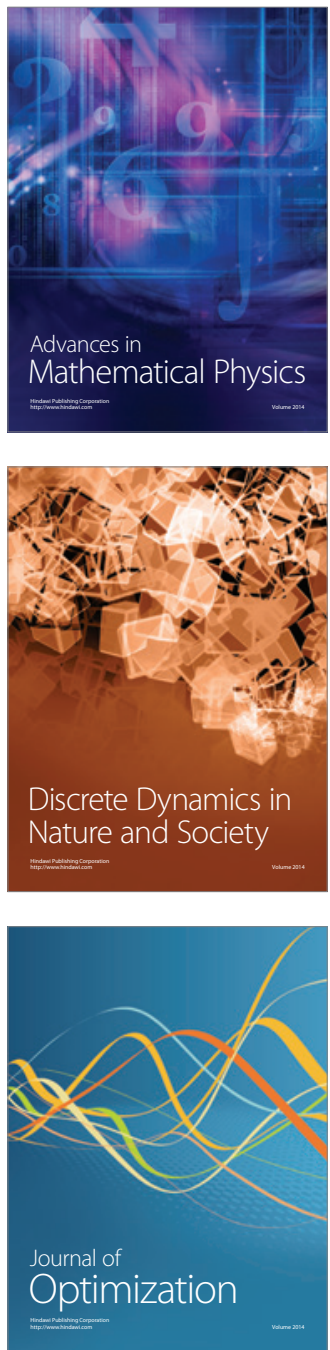